\title{
2005s-02 \\ Monte Carlo tests with nuisance parameters: \\ a general approach to finite-sample inference and non-standard asymptotics
}

Jean-Marie Dufour

\begin{tabular}{c}
\hline Série Scientifique \\
Scientific Series
\end{tabular}

Montréal

Août 2004

(publié en février 2005)

(C) 2005 Jean-Marie Dufour. Tous droits réservés. All rights reserved. Reproduction partielle permise avec citation du document source, incluant la notice (C).

Short sections may be quoted without explicit permission, iffull credit, including (C) notice, is given to the source.
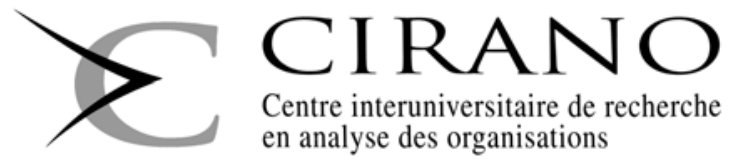

Centre interuniversitaire de recherche en analyse des organisations 


\section{CIRANO}

Le CIRANO est un organisme sans but lucratif constitué en vertu de la Loi des compagnies du Québec. Le financement de son infrastructure et de ses activités de recherche provient des cotisations de ses organisations-membres, d'une subvention d'infrastructure du Ministère du Développement économique et régional et de la Recherche, de même que des subventions et mandats obtenus par ses équipes de recherche.

CIRANO is a private non-profit organization incorporated under the Québec Companies Act. Its infrastructure and research activities are funded through fees paid by member organizations, an infrastructure grant from the Ministère du Développement économique et régional et de la Recherche, and grants and research mandates obtained by its research teams.

PARTENAIRE MAJEUR

$$
\text { Les organisations-partenaires / The Partner Organizations }
$$

. Ministère du Développement économique et régional et de la Recherche [MDERR]

PARTENAIRES

. Alcan inc.

. Axa Canada

. Banque du Canada

. Banque Laurentienne du Canada

. Banque Nationale du Canada

. Banque Royale du Canada

. Bell Canada

. BMO Groupe Financier

. Bombardier

. Bourse de Montréal

. Caisse de dépôt et placement du Québec

. Développement des ressources humaines Canada [DRHC]

. Fédération des caisses Desjardins du Québec

. GazMétro

. Groupe financier Norshield

. Hydro-Québec

. Industrie Canada

. Ministère des Finances du Québec

. Pratt \& Whitney Canada Inc.

. Raymond Chabot Grant Thornton

. Ville de Montréal

. École Polytechnique de Montréal

. HEC Montréal

. Université Concordia

. Université de Montréal

. Université du Québec

. Université du Québec à Montréal

. Université Laval

. Université McGill

. Université de Sherbrooke

ASSOCIE A :

. Institut de Finance Mathématique de Montréal $\left(\mathrm{IFM}^{2}\right)$

. Laboratoires universitaires Bell Canada

. Réseau de calcul et de modélisation mathématique $\left[\mathrm{RCM}^{2}\right]$

. Réseau de centres d'excellence MITACS (Les mathématiques des technologies de l'information et des systèmes complexes)

Les cahiers de la série scientifique $(\mathrm{CS})$ visent à rendre accessibles des résultats de recherche effectuée au CIRANO afin de susciter échanges et commentaires. Ces cahiers sont écrits dans le style des publications scientifiques. Les idées et les opinions émises sont sous l'unique responsabilité des auteurs et ne représentent pas nécessairement les positions du CIRANO ou de ses partenaires.

This paper presents research carried out at CIRANO and aims at encouraging discussion and comment. The observations and viewpoints expressed are the sole responsibility of the authors. They do not necessarily represent positions of CIRANO or its partners. 


\title{
Monte Carlo tests with nuisance parameters: a general approach to finite-sample inference and non-standard asymptotics
}

\author{
Jean-Marie Dufour ${ }^{\dagger}$
}

\begin{abstract}
Résumé / Abstract
La technique des tests de Monte Carlo ((MC; Dwass (1957), Barnard (1963)) constitue une méthode attrayante qui permet de construire des tests exacts fondés sur des statistiques dont la distribution exacte est difficile à calculer par des méthodes analytiques mais peut être simulée, pourvu que cette distribution ne dépende pas de paramètres de nuisance. Nous généralisons cette méthode dans deux directions: premièrement, en considérant le cas où le test de Monte Carlo est construit à partir de réplications échangeables d'une variable aléatoire dont la distribution peut comporter des discontinuités; deuxièmement, en étendant la méthode à des statistiques dont la distribution dépend de paramètres de nuisance (tests de Monte Carlo maximisés, MMC). Nous proposons aussi des versions simplifiées de la procédure MMC, qui ne sont valides qu'asymptotiquement mais fournissent néanmoins une méthode simple qui permet d'améliorer les approximations asymptotiques usuelles, en particulier dans des cas non standards (e.g., l'asymptotique en présence de racines unitaires). Nous montrons aussi que les tests basés sur la technique du bootstrap paramétrique peut s'interpréter comme une version simplifiée de la procédure MMC. Cette dernière fournit toutefois des tests asymptotiquement valides sous des conditions beaucoup plus générales que le bootstrap paramétrique.
\end{abstract}

Mots clés : test de Monte Carlo, test de Monte Carlo maximisé, test exact, test valide en échantillon fini, paramètre de nuisance, bornes, bootstrap, bootstrap paramétrique, recuit simulé, distribution asymptotique non standard.

\footnotetext{
* The author thanks Torben Andersen, Benoit Perron, Russell Davidson, V. P. Godambe, Joel Horowitz, Joanna Jasiak, Linda Khalaf, Jan Kiviet, James MacKinnon, Charles Manski, Eric Renault, Christopher Sims, Olivier Torrès, Pascale Valéry, Michael Veall, Mark Watson, and two anonymous referees for several useful comments. This work was supported by the Canada Research Chair Program (Chair in Econometrics, Université de Montréal), the Alexandervon-Humboldt Foundation (Germany), the Canadian Network of Centres of Excellence [program on Mathematics of Information Technology and Complex Systems (MITACS)], the Canada Council for the Arts (Killam Fellowship), the Natural Sciences and Engineering Research Council of Canada, the Social Sciences and Humanities Research Council of Canada, the Fonds de recherche sur la société et la culture (Québec), and the Fonds de recherche sur la nature et les technologies (Québec).

† Canada Research Chair Holder (Econometrics). Centre interuniversitaire de recherche en analyse des organisations (CIRANO), Centre interuniversitaire de recherche en économie quantitative (CIREQ), and Département de sciences économiques, Université de Montréal. Mailing address: Département de sciences économiques, Université de Montréal, C.P. 6128 succursale Centre-ville, Montréal, Québec, Canada H3C 3J7. Tel: 1 (514) 343 2400; Fax: 1 (514) 343 5831; e-mail: jean.marie.dufour@,umontreal.ca.
}

Web page: http://www.fas.umontreal.ca/SCECO/Dufour 
The technique of Monte Carlo (MC) tests [Dwass (1957), Barnard (1963)] provides an attractive method of building exact tests from statistics whose finite sample distribution is intractable but can be simulated (provided it does not involve nuisance parameters). We extend this method in two ways: first, by allowing for MC tests based on exchangeable possibly discrete test statistics; second, by generalizing the method to statistics whose null distributions involve nuisance parameters (maximized MC tests, MMC). Simplified asymptotically justified versions of the MMC method are also proposed and it is shown that they provide a simple way of improving standard asymptotics and dealing with nonstandard asymptotics (e.g., unit root asymptotics). Parametric bootstrap tests may be interpreted as a simplified version of the MMC method (without the general validity properties of the latter).

Keywords: Monte Carlo test, maximized monte Carlo test, finite sample test, exact test, nuisance parameter, bounds, bootstrap, parametric bootstrap, simulated annealing, asymptotics, nonstandard asymptotic distribution.

Codes JEL : C12; C15; C2; C52; C22 


\section{Contents}

List of Definitions, Propositions and Theorems iv

$\begin{array}{ll}\text { 1. Introduction } & 1\end{array}$

2. Monte Carlo tests without nuisance parameters 3

2.1. Monte Carlo tests based on statistics with continuous distributions . . . . . . 4

2.2. Monte Carlo tests based on general statistics . . . . . . . . . . . . . . 6

3. Power functions and concordance probabilities 9

4. Monte Carlo tests with nuisance parameters 11

5. Asymptotic Monte Carlo tests based on a consistent set estimator 16

6. Asymptotic Monte Carlo tests based on consistent point estimate 19

$\begin{array}{ll}\text { 7. Conclusion } & 22\end{array}$

A. Appendix: Proofs 24 


\section{List of Definitions, Propositions and Theorems}

2.1 Lemma : Distribution of ranks when ties have zero probability . . . . . . . . 5

2.2 Proposition : Validity of Monte Carlo tests when ties have zero probability . . . . 6

2.3 Lemma : Distribution of randomized ranks . . . . . . . . . . . . 7

2.4 Proposition : Validity of Monte Carlo tests for general statistics _ . . . . . . . . 8

4.1 Proposition : Validity of MMC tests when ties have zero probability . . . . . . . . . 14

4.2 Proposition : Validity of MMC tests for general statistics . . . . . . . . . . . . 15

5.1 Proposition : Asymptotic validity of confidence-set restricted MMC tests: continuous

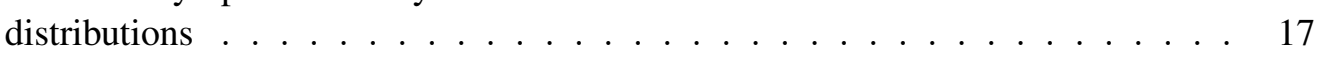

5.2 Proposition : Asymptotic validity of confidence-set restricted MMC tests: general distributions . . . . . . . . . . . . . . . . . . . . . 19

6.1 Proposition : Asymptotic validity of bootstrap $p$-values . . . . . . . . . . 21

Proof of Lemma $2.1 \ldots \ldots \ldots$

Proof of Proposition $2.2 \ldots \ldots \ldots \ldots$

Proof of Lemma $2.3 \ldots \ldots \ldots$

Proof of Proposition $2.4 \ldots \ldots \ldots \ldots$

Proof of Proposition $4.1 \ldots \ldots \ldots \ldots$

Proof of Proposition $4.2 \ldots \ldots \ldots \ldots \ldots$

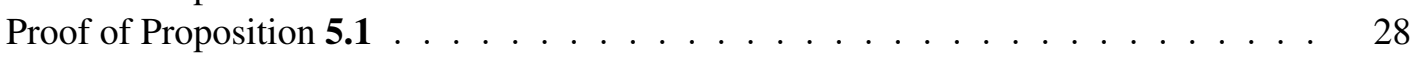

Proof of Proposition $5.2 \ldots \ldots \ldots \ldots \ldots \ldots$

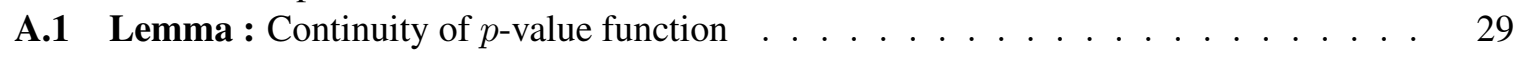

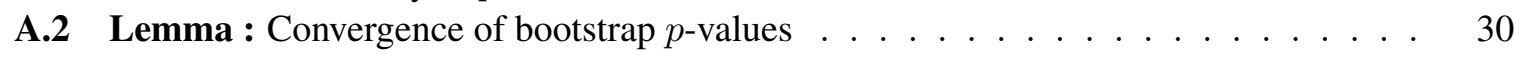

Proof of Proposition 6.1 . . . . . . . . . . . . . . . . . . 32 


\section{Introduction}

During the last twenty-five years, the development of faster and cheaper computers has made Monte Carlo techniques more affordable and attractive in statistical analysis. In particular, such techniques may now be used routinely for data analysis. Important developments in this area include the use of bootstrap techniques for improving standard asymptotic approximations [for reviews, see Efron (1982), Beran and Ducharme (1991), Efron and Tibshirani (1993), Hall (1992), Jeong and Maddala (1993), Vinod (1993), Shao and Tu (1995), Davison and Hinkley (1997), Chernick (1999) and Horowitz (1997)] and techniques where estimators and forecasts are obtained from criteria evaluated by simulation [see McFadden (1989), Mariano and Brown (1993), Hajivassiliou (1993), Keane (1993), Gouriéroux and Monfort (1996) and Gallant and Tauchen (1996)].

With respect to tests and confidence sets, these techniques only have asymptotic justifications and do not yield inferences that are provably valid (in the sense of correct levels) in finite samples. Here it is of interest to note that the use of simulation in the execution of tests was suggested much earlier than recent bootstrap and simulation-based techniques. For example, randomized tests have been proposed long ago as a way of obtaining tests with any given level from statistics with discrete distributions (e.g., sign and rank tests); see Lehmann (1986). A second interesting possibility is the technique of Monte Carlo tests originally suggested by Dwass (1957) for implementing permutation tests and later extended by Barnard (1963), Hope (1968) and Birnbaum (1974). This technique has the great attraction of providing exact (randomized) tests based on any statistic whose finite sample distribution may be intractable but can be simulated. The validity of the tests so obtained does not depend at all on the number of replications made (which can be small). Only the power of the procedure is influenced by the number of replications, but the power gains associated with lengthy simulations are typically rather small. For further discussion of Monte Carlo tests, see Besag and Diggle (1977), Dufour and Kiviet (1996, 1998), Edgington (1980), Edwards (1985), Edwards and Berry (1987), Foutz (1980), Jöckel (1986), Kiviet and Dufour (1997), Marriott (1979) and Ripley (1981).

An important limitation of the technique of Monte Carlo tests is the fact that one needs to have a statistic whose distribution does not depend on nuisance parameters. This obviously limits considerably its applicability. The main objective of this paper is to extend the technique of Monte Carlo tests in order to allow for the presence of nuisance parameters in the null distribution of the test statistic.

In Section 2, we summarize and extend results on Monte Carlo (MC) tests when the null distribution of a test statistic does not involve nuisance parameters. In particular, we put them in a form that will make their extension to cases with nuisance parameters easy and intuitive, and we generalize them by allowing for MC tests based on exchangeable (possibly non independent) replications and statistics with discrete distributions. These generalizations allow, in particular, for various nonparametric tests (e.g., permutation tests) as well as test statistics where certain parameters are themselves evaluated by simulation. We deal with possibly discrete (or mixtures of continuous and discrete distributions) by exploiting Hájek's (1969) method of randomized ranks for breaking ties in rank tests, which is both simple to implement and allows one to easily deal with exchangeable (as opposed to i.i.d.) simulations. On the problem of discrete distributions, it is also of interest that the method 
proposed by Jöckel (1986) was derived under the assumption of i.i.d. MC replications.

In Section 3, we study how the power of Monte Carlo tests is related to the number of replications used and the sensitivity of the conclusions to the randomized nature of the procedure. In particular, given the observed (randomized) $p$-value of the Monte Carlo test, we see that the probability of an eventual reversal of the conclusion of the procedure (rejection or acceptance at a given level, e.g. $5 \%$ ) can easily be computed.

In Section 4, we present the extension to statistics whose null distribution depends on nuisance parameters. This procedure is based on considering a simulated $p$-value function which depends on nuisance parameters (under the null hypothesis). We show that maximizing the latter with respect to the nuisance parameters yields a test with provably exact level, irrespective of the sample size and the number replications used. For this reason, we call the latter maximized Monte Carlo (MMC) tests. As one would expect for a statistic whose distribution depends on unknown nuisance parameters, the probability of type I error for a MMC test can be lower (but not higher) than the level of the test and the procedure can be conservative. We also discuss how this maximization can be achieved in practice, e.g. through simulated annealing techniques.

In the two next sections, we discuss simplified (asymptotically justified) approximate versions of the proposed procedures, which involve the use of consistent set or point estimates of model parameters. In Section 5, we suggest a method [the consistent set estimate MMC method (CSEMMC)] which is applicable when a consistent set estimator of the nuisance parameters [e.g., a random subset of the parameter space whose probability of covering the nuisance parameters converges to one as the sample size goes to infinity] is available. The approach proposed involves maximizing the simulated $p$-value function over the consistent set estimate, as opposed to the full nuisance parameter space. This procedure may thus be computationally much less costly. Using a consistent set estimator (or confidence set), as opposed to a point estimate, to deal with nuisance parameters is especially useful because it allows one to obtain asymptotically valid tests even when the test statistic does not converge in distribution or when the asymptotic distribution depends on nuisance parameters possibly in a discontinuous way. Consequently, there is no need to study the asymptotic distribution of the test statistic considered or even to establish its existence. ${ }^{1}$ This consistent set estimator MMC method (CSEMMC) may be viewed as an asymptotic Monte Carlo extension of finite-sample twostage procedures proposed in Dufour (1990), Dufour and Kiviet (1996, 1998), Campbell and Dufour (1997), and Dufour, Hallin and Mizera (1998). These features may be contrasted with those of bootstrap methods which can fail to provide asymptotically valid tests when the test statistic simulated has an asymptotic distribution involving nuisance parameters, especially if the asymptotic distribution has discontinuities with respect to the nuisance parameters [see Athreya (1987), Basawa, Mallik, McCormick, Reeves and Taylor (1991) and Sriram (1994), Andrews (2000), Benkwitz, Lütkepohl and Neumann (2000), Inoue and Kilian (2002, 2003)].

In Section 6, we consider the simplest form of a Monte Carlo test with nuisance parameters, i.e. the one where the consistent set estimate has been replaced by a consistent point estimate. In other words, the distribution of the test statistic is simulated after replacing the nuisance parameters

\footnotetext{
${ }^{1} \mathrm{~A}$ case where the distribution of a test statistic does not converge in distribution is the one where the associated sequence of distribution functions has several accumulation points, allowing different subsequences to have different limiting distributions.
} 
by a consistent point estimate. Such a procedure can be interpreted as a parametric bootstrap test based on the percentile method [see Efron and Tibshirani (1993, Chapter 16) and Hall (1992)]. The term "parametric" may however be misleading here, because such MC tests can be applied as well to nonparametric (distribution-free) test statistics. We give general conditions under which a Monte Carlo test obtained after replacing an unknown nuisance parameter yield an asymptotically valid test in cases where the limit distribution of the test statistic involves nuisance parameters. Following the general spirit of Monte Carlo testing and in contrast with typical bootstrap arguments, the proofs take the number of Monte Carlo simulations as fixed (possibly very small, such as 19 to obtain a test with level 0.05). As in standard bootstrap arguments, the conditions considered involve a smooth (continuous) dependence of the asymptotic distribution upon the nuisance parameters. It is, however, important to note that these conditions are more restrictive and more difficult to check than those under which CSEMMC procedures would be applicable. We conclude in Section 7.

\section{Monte Carlo tests without nuisance parameters}

Let us consider a family of probability spaces $\left\{\left(\mathcal{Z}, \mathcal{A}_{\mathcal{Z}}, \mathrm{P}_{\theta}\right): \theta \in \Omega\right\}$, where $\mathcal{Z}$ is a sample space, $\mathcal{A}_{\mathcal{Z}}$ a $\sigma$-algebra of subsets of $\mathcal{Z}$, and $\Omega$ a parameter space (possible infinite dimensional). Let also $S \equiv S(\omega), \omega \in \mathcal{Z}$, be a real-valued $\mathcal{A}_{\mathcal{Z}}$-measurable function whose distribution is determined by $\mathrm{P}_{\theta_{0}}$ - i.e., $\theta_{0}$ is the "true" parameter vector. We wish to test the hypothesis

$$
H_{0}: \theta_{0} \in \Omega_{0}
$$

where $\Omega_{0}$ is a nonempty subset of $\Omega$, using a critical region of the form $\{S \geq c\}$. Although, in general, the distribution of $S$ under $H_{0}$ depends on the unknown value of $\theta_{0}$, we shall assume in this section that this distribution does not depend on (unknown) nuisance parameters so that we can write

$$
\mathrm{P}_{\theta}[S \leq x]=F(x), \quad \text { for all } \theta \in \Omega_{0},
$$

where $F(x)$ is the unique distribution that $S$ can have under $H_{0}$. In view of this assumption, we shall - until further notice - compute probabilities under the (unique) $\mathrm{P} \equiv \mathrm{P}_{\theta_{0}}$ when $\theta_{0} \in \Omega_{0}$. The constant $c$ is chosen so that

$$
\mathrm{P}[S \geq c]=1-F(c)+\mathrm{P}[S=c] \leq \alpha
$$

where $\alpha$ is the desired level of the test $(0<\alpha<1)$. Note that the critical region $S \geq c$ can also be put in two useful alternative forms, which are equivalent to $S \geq c$ with probability one (i.e., they can differ from the critical region $S \geq c$ only on a set of zero probability):

$$
\begin{gathered}
G(S) \leq G(c), \\
S \geq F^{-1}\left[(F(c)-\mathrm{P}[S=c])^{+}\right]=F^{-1}\left[(1-G(c))^{+}\right]
\end{gathered}
$$

where

$$
G(x)=\mathrm{P}[S \geq x]=1-F(x)+\mathrm{P}[S=x]
$$


is the "tail area" or " $p$-value function" associated with $F$, and $F^{-1}$ is the quantile function of $F$, with the conventions

$$
F^{-1}\left(q^{+}\right)=\lim _{\varepsilon \downarrow 0} F^{-1}(q+\varepsilon)=\inf \left\{F^{-1}\left(q_{0}\right): q_{0}>q\right\}, 0 \leq q \leq 1,
$$

$F^{-1}\left(1^{+}\right)=\infty$ and $F^{-1}\left(0^{+}\right)=F^{-1}(0)$. For any probability distribution function $F(x)$, the quantile function $F^{-1}(q)$ is defined as follows:

$$
\begin{array}{rlrl}
F^{-1}(q) & =\inf \{x: F(x) \geq q\}, & & \text { if } 0<q<1, \\
& =\inf \{x: F(x)>0\}, & & \text { if } q=0 \\
& =\sup \{x: F(x)<1\}, & \text { if } q=1
\end{array}
$$

see Reiss (1989, p. 13). In general, $F^{-1}(q)$ takes its values in the extended real numbers $\overline{\mathbb{R}}=$ $\mathbb{R} \cup\{-\infty,+\infty\}$ and, for coherence, we set $F(-\infty)=0$ and $F(\infty)=1$. Using (2.7), it is easy to see that

$$
F^{-1}\left[(F(c)-\mathrm{P}[S=c])^{+}\right]=c
$$

when: $0<F(c)<1$ and either for $\mathrm{P}(S=c)>0$ or $F(x)$ is continuous and strictly monotonic in an open neighborhood containing $c$. However, the formulation (2.5) remains valid in all cases.

\subsection{Monte Carlo tests based on statistics with continuous distributions}

Consider now a situation where the distribution of $S$ under $H_{0}$ may not be easy to compute analytically but can be simulated. Let $S_{1}, \ldots, S_{N}$ be a sample of identically distributed real random variables with the same distribution as $S$. Typically, it is assumed that $S_{1}, \ldots, S_{N}$ are also independent. However, we will observe that the exchangeability of $S_{1}, \ldots, S_{N}$ is sufficient for most of the results presented below. ${ }^{2}$ This can accommodate a wide array of situations, where the simulated statistics are not independent because they involve common (conditioning) variables, such as: statistics obtained by permuting randomly a given set of observations (permutation tests), tests which are simulated conditionally on a common set of initial values (e.g., in time series models), common regressors or a common subsample [see the Anderson-Rubin-type split-sample test described in Dufour and Jasiak (2001)], tests that depend on a common independent simulation [e.g., tests based on a criterion evaluated by a preliminary simulation, such as the simulated method of moments or indirect inference; see Gouriéroux and Monfort (1996)], etc.

The technique of Monte Carlo tests provides a simple method allowing one to replace the theoretical distribution $F(x)$ by its sample analogue based on $S_{1}, \ldots, S_{N}$ :

$$
\hat{F}_{N}(x) \equiv \hat{F}_{N}[x ; S(N)]=\frac{1}{N} \sum_{i=1}^{N} \mathbf{1}\left(S_{i} \leq x\right)
$$

\footnotetext{
${ }^{2}$ The elements of a random vector $\left(S_{1}, S_{2}, \ldots, S_{N}\right)^{\prime}$ are exchangeable (or P-exchangeable) if $\left(S_{r_{1}}, S_{r_{2}}, \ldots, S_{r_{N}}\right)^{\prime} \sim\left(S_{1}, S_{2}, \ldots, S_{N}\right)^{\prime}$ for any permutation $\left(r_{1}, r_{2}, \ldots, r_{N}\right)$ of the integers $(1,2, \ldots, N)$ under the relevant probability measure $P$.
} 
where $S(N)=\left(S_{1}, \ldots, S_{N}\right)^{\prime}$ and $\mathbf{1}(C)$ is the indicator function associated with the condition $C$ :

$$
\begin{aligned}
\mathbf{1}(C) & =1, \text { if condition } C \text { holds, } \\
& =0, \text { otherwise }
\end{aligned}
$$

In the latter notation, $C$ may also be replaced by an event $A \in \mathcal{A}_{\mathcal{Z}}$, in which case $\mathbf{1}(A) \equiv \mathbf{1}(\omega \in A)$ where $\omega$ is the element drawn from the sample space.

We also consider the corresponding sample tail area (or survival) function:

$$
\hat{G}_{N}[x ; S(N)]=\frac{1}{N} \sum_{i=1}^{N} \mathbf{1}\left(S_{i} \geq x\right) .
$$

The sample distribution function is related to the ranks $R_{1}, \ldots, R_{N}$ of the variables $S_{1}, \ldots, S_{N}$ (when put in ascending order) by the expression:

$$
R_{j}=N \hat{F}_{N}\left[S_{j} ; S(N)\right]=\sum_{i=1}^{N} \mathbf{1}\left(S_{i} \leq S_{j}\right), \quad j=1, \ldots, N .
$$

The central property we shall exploit here is the following: to obtain critical values or compute $p$-values, the "theoretical" null distribution $F(x)$ can be replaced by its simulation-based "estimate" $\hat{F}_{N}(x)$ in a way that will preserve the level of the test in finite samples, irrespective of the number $N$ of replications used. For continuous distributions, this property is expressed by Proposition $\mathbf{2 . 2}$ below, which is easily proved by using the following simple lemma.

Lemma 2.1 Distribution OF RANKS WHEN TIES HAVE ZERO PROBABILITY. Let $\left(y_{1}, \ldots, y_{N}\right)^{\prime}$ be a $N \times 1$ vector of $\mathrm{P}$-exchangeable real random variables such that

$$
\mathrm{P}\left(y_{i}=y_{j}\right)=0 \quad \text { for } i \neq j, i, j=1, \ldots, N,
$$

and let $R_{j}=\sum_{i=1}^{N} \mathbf{1}\left(y_{i} \leq y_{j}\right)$ be the rank of $y_{j}$ when $y_{1}, \ldots, y_{N}$ are ranked in nondecreasing $\operatorname{order}(j=1, \ldots, N)$. Then, for $j=1, \ldots, N$,

$$
\begin{aligned}
\mathrm{P}\left(R_{j} / N\right. & \leq x)=I[x N] / N, & & \text { for } 0 \leq x \leq 1, \\
\mathrm{P}\left(R_{j} / N \geq x\right) & =1, & & \text { if } x \leq 0, \\
& =(I[(1-x) N]+1) / N, & & \text { if } 0<x \leq 1, \\
& =0, & & \text { if } x>1,
\end{aligned}
$$

where $I[x]$ is the largest integer less than or equal to $x$.

Note that we use the symbol $I[x]$ rather than the common notation $[x]$ to represent the integer part of a number $x$, because we use heavily brackets elsewhere in the paper, so that the notation $[\cdot]$ could lead to confusions. It is clear that condition (2.12) is satisfied whenever the variables $y_{1}, \ldots, y_{N}$ are independent with continuous distribution functions [see Hájek (1969, pp. 20-21)], 
or when the vector $\left(y_{1}, \ldots, y_{N}\right)^{\prime}$ has an absolutely continuous distribution (with respect to the Lebesgue measure on $\mathbb{R}^{N}$ ).

Proposition 2.2 VAlidity of Monte Carlo tests When ties haVe Zero Probability. Let $\left(S_{0}, S_{1}, \ldots, S_{N}\right)^{\prime}$ be a $(N+1) \times 1$ vector of exchangeable real random variables such that

$$
\mathrm{P}\left(S_{i}=S_{j}\right)=0 \quad \text { for } i \neq j, i, j=0,1, \ldots, N
$$

let $\hat{F}_{N}(x) \equiv \hat{F}_{N}[x ; S(N)], \hat{G}_{N}(x)=\hat{G}_{N}[x ; S(N)]$ and $\hat{F}_{N}^{-1}(x)$ be defined as in (2.7) - (2.10), and set

$$
\hat{p}_{N}(x)=\frac{N \hat{G}_{N}(x)+1}{N+1} .
$$

Then,

$$
\begin{gathered}
\mathrm{P}\left[\hat{G}_{N}\left(S_{0}\right) \leq \alpha_{1}\right]=\mathrm{P}\left[\hat{F}_{N}\left(S_{0}\right) \geq 1-\alpha_{1}\right]=\frac{I\left[\alpha_{1} N\right]+1}{N+1}, \quad \text { for } 0 \leq \alpha_{1} \leq 1, \\
\mathrm{P}\left[S_{0} \geq \hat{F}_{N}^{-1}\left(1-\alpha_{1}\right)\right]=\frac{I\left[\alpha_{1} N\right]+1}{N+1}, \quad \text { for } 0<\alpha_{1}<1,
\end{gathered}
$$

and

$$
\mathrm{P}\left[\hat{p}_{N}\left(S_{0}\right) \leq \alpha\right]=\frac{I[\alpha(N+1)]}{N+1}, \quad \text { for } 0 \leq \alpha \leq 1 .
$$

The latter proposition can be used as follows: choose $\alpha_{1}$ and $N$ so that

$$
\alpha=\frac{I\left[\alpha_{1} N\right]+1}{N+1}
$$

is the desired significance level. Provided $N$ is reasonably large, $\alpha_{1}$ will be very close to $\alpha$; in particular, if $\alpha(N+1)$ is an integer, we can take $\alpha_{1}=\alpha-((1-\alpha) / N)$, in which case we see easily that the critical region $\hat{G}_{N}\left(S_{0}\right) \leq \alpha_{1}$ is equivalent to $\hat{G}_{N}\left(S_{0}\right)<\alpha$. For $0<\alpha<1$, the randomized critical region $S_{0} \geq \hat{F}_{N}^{-1}\left(1-\alpha_{1}\right)$ has the same level $(\alpha)$ as the nonrandomized critical region $S_{0} \geq F^{-1}(1-\alpha)$, or equivalently the critical regions $\hat{p}_{N}\left(S_{0}\right) \leq \alpha$ and $\hat{G}_{N}\left(S_{0}\right) \leq \alpha_{1}$ have the same level as the critical region $G\left(S_{0}\right) \equiv 1-F\left(S_{0}\right) \leq \alpha$.

\subsection{Monte Carlo tests based on general statistics}

The assumption (2.15), which states that ties have zero probability, plays an important role in proving Proposition 2.2. However, it is possible to prove analogous results for general sequences of exchangeable random variables (which may exhibit ties with positive probability), provided we consider a properly randomized empirical distribution function. For this purpose, we introduce randomized ranks which are obtained like ordinary ranks except that ties are "broken" according to a uniform distribution. More precisely, let us associate with each variable $S_{j}, j=1, \ldots, N$, a 
random variable $U_{j}, j=1, \ldots, N$ such that

$$
U_{1}, \ldots, U_{N} \stackrel{i . i . d .}{\sim} U(0,1)
$$

$U(N)=\left(U_{1}, \ldots, U_{N}\right)^{\prime}$ is independent of $S(N)=\left(S_{1}, \ldots, S_{N}\right)^{\prime}$ where $U(0,1)$ is the uniform distribution on the interval $(0,1)$. Then, we consider the pairs

$$
Z_{j}=\left(S_{j}, U_{j}\right), \quad j=1, \ldots, N,
$$

which are ordered according to the lexicographic order:

$$
\left(S_{i}, U_{i}\right) \leq\left(S_{j}, U_{j}\right) \Longleftrightarrow\left\{S_{i}<S_{j} \text { or }\left(S_{i}=S_{j} \text { and } U_{i} \leq U_{j}\right)\right\}
$$

Using the indicator

$$
\mathbf{1}\left[\left(x_{1}, u_{1}\right) \leq\left(x_{2}, u_{2}\right)\right]=\mathbf{1}\left(x_{1}<x_{2}\right)+\delta\left(x_{1}-x_{2}\right) \mathbf{1}\left(u_{1} \leq u_{2}\right),
$$

$S_{1}, \ldots, S_{N}$ are then ordered like the pairs $Z_{1}, \ldots, Z_{N}$ according to (2.23), which yield "randomized ranks":

$$
\tilde{R}_{j}[S(N), U(N)]=\sum_{i=1}^{N} \mathbf{1}\left[\left(S_{i}, U_{i}\right) \leq\left(S_{j}, U_{j}\right)\right],
$$

$j=1, \ldots, N$. By the continuity of the uniform distribution, the ranks $\tilde{R}_{j}=\tilde{R}_{j}[S(N), U(N)]$, $j=1, \ldots, N$, are all distinct with probability 1 , so that the randomized rank vector $\left(\tilde{R}_{1}, \tilde{R}_{2}, \ldots, \tilde{R}_{N}\right)^{\prime}$ is a permutation of $(1,2, \ldots, N)^{\prime}$ with probability 1 . Furthermore when $S_{j} \neq S_{i}$ for all $j \neq i$, we have $\tilde{R}_{j}=R_{j}$ : provided (2.15) holds, we have $\tilde{R}_{j}=R_{j}, j=1, \ldots, N$, with probability 1 [where $R_{j}$ is defined in (2.11)]. We can now state the following extension of Lemma 2.1 .

Lemma 2.3 DistribUtion OF RANDOMIZED RANKS. Let $y(N)=\left(y_{1}, \ldots, y_{N}\right)^{\prime}$ be a $N \times 1$ vector of exchangeable real random variables and let $\tilde{R}_{j}=\tilde{R}_{j}[y(N), U(N)]$ be defined as in (2.25) where $U(N)=\left(U_{1}, \ldots, U_{N}\right)^{\prime}$ is a vector of i.i.d. $U(0,1)$ variables independent of $y(N)$. Then, for $j=1, \ldots, N$,

$$
\begin{array}{rlrl}
\mathrm{P}\left(\tilde{R}_{j} / N \leq x\right)=I[x N] / N, & \text { for } 0 \leq x \leq 1, \\
\mathrm{P}\left(\tilde{R}_{j} / N \geq x\right) & =1, & & \text { if } x \leq 0, \\
& =(I[(1-x) N]+1) / N, & & \text { if } 0<x \leq 1, \\
& =0, & & \text { if } x>1 .
\end{array}
$$

To the above randomized rankings, it is natural to associate the following randomized empirical (pseudo-)distribution function:

$$
\tilde{F}_{N}\left[x ; U_{0}, S(N), U(N)\right]=\frac{1}{N} \sum_{i=1}^{N} \mathbf{1}\left[\left(S_{i}, U_{i}\right) \leq\left(x, U_{0}\right)\right]
$$




$$
=1-\hat{G}_{N}[x ; S(N)]+T_{N}\left[x ; U_{0}, S(N), U(N)\right]
$$

where $U_{0}$ is a $U(0,1)$ random variable independent of $S(N)$ and $U(N)$,

$$
T_{N}\left[x ; U_{0}, S(N), U(N)\right]=\frac{1}{N} \sum_{i=1}^{N} \delta\left(S_{i}-x\right) \mathbf{1}\left(U_{i} \leq U_{0}\right)=\frac{1}{N} \sum_{i \in E_{N}(x)} \mathbf{1}\left(U_{i} \leq U_{0}\right)
$$

and $E_{N}(x)=\left\{i: S_{i}=x, 1 \leq i \leq N\right\}$. The function $\tilde{F}_{N}[x ; \cdot]$ retains all the properties of a probability distribution function, except for the fact that it may not be right continuous at some of its jump points (where it may take values between its right and left limits). We can also define the corresponding tail area function:

$$
\begin{aligned}
& \tilde{G}_{N}\left[x ; U_{0}, S(N), U(N)\right]=\frac{1}{N} \sum_{i=1}^{N} \mathbf{1}\left[\left(S_{i}, U_{i}\right) \geq\left(x, U_{0}\right)\right] \\
&=1-\hat{F}_{N}[x ; S(N)]+\bar{T}_{N}\left[x ; U_{0}, S(N), U(N)\right], \\
& \bar{T}_{N}\left[x ; U_{0}, S(N), U(N)\right]=\frac{1}{N} \sum_{i=1}^{N} \delta\left(S_{i}-x\right) \mathbf{1}\left(U_{i} \geq U_{0}\right)=\frac{1}{N} \sum_{i \in E_{N}(x)} \mathbf{1}\left(U_{i} \geq U_{0}\right) .
\end{aligned}
$$

From (2.28) - (2.31), we see that the following inequalities must hold:

$$
\begin{aligned}
& 1-\hat{G}_{N}[x ; S(N)] \leq \tilde{F}_{N}\left[x ; U_{0}, S(N), U(N)\right] \leq \hat{F}_{N}[x ; S(N)], \\
& 1-\hat{F}_{N}[x ; S(N)] \leq \tilde{G}_{N}\left[x ; U_{0}, S(N), U(N)\right] \leq \hat{G}_{N}[x ; S(N)] .
\end{aligned}
$$

When no element of $S(N)$ is equal to $x$ [i.e., when $E_{N}(x)$ is empty], we have:

$$
\begin{aligned}
\tilde{G}_{N}\left[x ; U_{0}, S(N), U(N)\right] & =\hat{G}_{N}[x ; S(N)]=1-\hat{F}_{N}[x ; S(N)] \\
& =1-\tilde{F}_{N}\left[x ; U_{0}, S(N), U(N)\right] .
\end{aligned}
$$

Using the above observations, it is then easy to establish the following proposition.

Proposition 2.4 VAlidity of Monte CARlo tests for general statistics. Let $\left(S_{0}, S_{1}, \ldots, S_{N}\right)^{\prime}$ be a $(N+1) \times 1$ vector of exchangeable real random variables, let $\left(U_{0}, U_{1}, \ldots, U_{N}\right)^{\prime}$ be a $(N+1) \times 1$ vector of i.i.d. $U(0,1)$ random variables independent of $\left(S_{0}, S_{1}, \ldots, S_{N}\right)^{\prime}$, let $\hat{F}_{N}(x) \equiv \hat{F}_{N}[x ; S(N)], \hat{G}_{N}(x) \equiv \hat{G}_{N}[x ; S(N)], \tilde{F}_{N}(x) \equiv$ $\tilde{F}_{N}\left[x ; U_{0}, S(N), U(N)\right]$ and $\tilde{G}_{N}(x) \equiv \tilde{G}_{N}\left[x ; U_{0}, S(N), U(N)\right]$ be defined as in (2.8) (2.10) and (2.28) - (2.30), with $S(N)=\left(S_{1}, \ldots, S_{N}\right)$ and $U(N)=\left(U_{1}, \ldots, U_{N}\right)$, and let

$$
\tilde{p}_{N}(x)=\frac{N \tilde{G}_{N}(x)+1}{N+1} .
$$


Then for $0 \leq \alpha_{1} \leq 1$,

$$
\begin{aligned}
\mathrm{P}\left[\hat{G}_{N}\left(S_{0}\right) \leq \alpha_{1}\right] & \leq \mathrm{P}\left[\tilde{G}_{N}\left(S_{0}\right) \leq \alpha_{1}\right]=\mathrm{P}\left[\tilde{F}_{N}\left(S_{0}\right) \geq 1-\alpha_{1}\right] \\
& =\frac{I\left[\alpha_{1} N\right]+1}{N+1} \leq \mathrm{P}\left[\hat{F}_{N}\left(S_{0}\right) \geq 1-\alpha_{1}\right]
\end{aligned}
$$

with $\mathrm{P}\left[\hat{F}_{N}\left(S_{0}\right) \geq 1-\alpha_{1}\right]=\mathrm{P}\left[S_{0} \geq \hat{F}_{N}^{-1}\left(1-\alpha_{1}\right)\right]$ for $0<\alpha_{1}<1$, and defining $\hat{p}_{N}(x)$ as in (2.16),

$$
\mathrm{P}\left[\hat{p}_{N}\left(S_{0}\right) \leq \alpha\right] \leq \mathrm{P}\left[\tilde{p}_{N}\left(S_{0}\right) \leq \alpha\right]=\frac{I[\alpha(N+1)]}{N+1}, \text { for } 0 \leq \alpha \leq 1 .
$$

In view of the fact that $\tilde{G}_{N}\left(S_{0}\right)=\hat{G}_{N}\left(S_{0}\right)$ with probability one when the zero probability tie condition [i.e. (2.15)] holds, it is straightforward to see that Proposition 2.2 is entailed by Proposition 2.4 .

\section{Power functions and concordance probabilities}

The procedures described above are randomized in the sense that the result of the tests depend on auxiliary simulations. This raises the issue of the sensitivity of the results to these simulations. To study this more closely, let us suppose that:

$$
\begin{aligned}
& S_{0}, S_{1}, \ldots, S_{N} \text { are independent with } \\
& \mathrm{P}\left(S_{i} \leq x\right)=F(x), \mathrm{P}\left(S_{i} \geq x\right)=G(x), \mathrm{P}\left(S_{i}=x\right)=g(x), i=1, \ldots, N, \\
& \mathrm{P}\left(S_{0} \leq x\right)=H(x), \mathrm{P}\left(S_{0} \geq x\right)=K(x) .
\end{aligned}
$$

Then, it is easy to see that $N \tilde{G}_{N}(x)$ follows a binomial distribution $\operatorname{Bi}(N, p)$ with number of trials $N$ and probability of "success" $p=\bar{G}(x, u)$, where

$$
\begin{aligned}
\bar{G}(x, u) & =\mathrm{P}\left(\mathbf{1}\left[\left(S_{i}, U_{i}\right) \geq(x, u)\right]=1\right)=\mathrm{P}\left(S_{i}>x\right)+\mathrm{P}\left(S_{i}=x\right) \mathrm{P}\left(U_{i} \geq u\right) \\
& =1-F(x)+g(x)(1-u)
\end{aligned}
$$

and we can compute the conditional probability given $\left(S_{0}, U_{0}\right)$ of the critical region $\tilde{G}_{N}\left(S_{0}\right) \leq \alpha_{1}$ :

$$
\begin{aligned}
\mathrm{P}\left[\tilde{G}_{N}\left(S_{0}\right) \leq \alpha_{1} \mid\left(S_{0}, U_{0}\right)\right] & =\mathrm{P}\left[\sum_{i=1}^{N} \mathbf{1}\left[\left(S_{i}, U_{i}\right) \geq\left(S_{0}, U_{0}\right)\right] \leq I\left[\alpha_{1} N\right] \mid\left(S_{0}, U_{0}\right)\right] \\
& =\sum_{k=0}^{I\left[\alpha_{1} N\right]}\left(\begin{array}{l}
N \\
k
\end{array}\right) \bar{G}\left(S_{0}, U_{0}\right)^{k}\left[1-\bar{G}\left(S_{0}, U_{0}\right)\right]^{N-k}
\end{aligned}
$$


where $\left(\begin{array}{l}N \\ k\end{array}\right)=N ! /[k !(N-k) !]$. Similarly, we can also write:

$$
\mathrm{P}\left[\hat{G}_{N}\left(S_{0}\right) \leq \alpha_{1} \mid S_{0}\right]=\sum_{k=0}^{I\left[\alpha_{1} N\right]}\left(\begin{array}{l}
N \\
k
\end{array}\right) G\left(S_{0}\right)^{k}\left(1-G\left(S_{0}\right)\right)^{N-k}
$$

When $F(x)$ is continuous, so that $g(x)=0$, we have:

$$
\begin{aligned}
\mathrm{P}\left[\tilde{G}_{N}\left(S_{0}\right) \leq \alpha_{1} \mid\left(S_{0}, U_{0}\right)\right] & =\mathrm{P}\left[\hat{G}_{N}\left(S_{0}\right) \leq \alpha_{1} \mid S_{0}\right] \\
& =\sum_{k=0}^{I\left[\alpha_{1} N\right]}\left(\begin{array}{l}
N \\
k
\end{array}\right)\left[1-F\left(S_{0}\right)\right]^{k} F\left(S_{0}\right)^{N-k} .
\end{aligned}
$$

Using (3.3), we can find a close-form expression for the power of the randomized test $\tilde{G}\left(S_{0}\right) \leq$ $\alpha_{1}$ for any null hypothesis which entails that $S_{0}$ has the distribution $F(\cdot)$ against an alternative under which its distribution is $H(\cdot)$ :

$$
\begin{aligned}
\mathrm{P}\left[\tilde{G}_{N}\left(S_{0}\right) \leq \alpha_{1}\right] & =\underset{\left(S_{0}, U_{0}\right)}{\mathrm{E}}\left\{\mathrm{P}\left[\tilde{G}_{N}\left(S_{0}\right) \leq \alpha_{1} \mid\left(S_{0}, U_{0}\right)\right]\right\} \\
& =\sum_{k=0}^{I\left[\alpha_{1} N\right]}\left(\begin{array}{l}
N \\
k
\end{array}\right) \iint_{0}^{1} \bar{G}(x, u)^{k}[1-\bar{G}(x, u)]^{N-k} d u d H(x) .
\end{aligned}
$$

Furthermore, when $F(x)$ is continuous everywhere, the latter expression simplifies and we can write:

$$
\begin{aligned}
\mathrm{P}\left[\tilde{G}_{N}\left(S_{0}\right) \leq \alpha_{1}\right] & =\mathrm{P}\left[\hat{G}_{N}\left(S_{0}\right) \leq \alpha_{1}\right] \\
& =\sum_{k=0}^{I\left[\alpha_{1} N\right]}\left(\begin{array}{l}
N \\
k
\end{array}\right) \int[1-F(x)]^{k} F(x)^{N-k} d H(x) .
\end{aligned}
$$

The above formulae will be useful in establishing the validity of simplified asymptotic Monte Carlo tests in the presence of nuisance parameters. They also allow one to compute the probability that the result of the randomized test $\tilde{G}_{N}\left(S_{0}\right) \leq \alpha_{1}$ be different of the result of the corresponding nonrandomized test $G\left(S_{0}\right) \leq \alpha \equiv\left(\left[N \alpha_{1}\right]+1\right) /(N+1)$. For example, let $\hat{\alpha}_{0}=G\left(S_{0}\right)$ the " $p$-value" one would obtain if the function $G(x)$ were easy to compute (the $p$-value of the "fundamental test"). The latter is generally different from the $p$-value $\tilde{p}_{N}\left(S_{0}\right)$ or $\hat{p}_{N}\left(S_{0}\right)$ obtained from a Monte Carlo test based on $S_{1}, \ldots, S_{N}$. An interesting question here is the probability that the Monte Carlo test yields a conclusion different from the one based on $\hat{\alpha}_{0}$. To study this, we shall consider the test which rejects the null hypothesis $H_{0}$ when $\hat{p}_{N}\left(S_{0}\right) \leq \alpha$ under the assumptions (3.1).

If $\hat{\alpha}_{0}>\alpha$ (in which case $H_{0}$ is not rejected at level $\alpha$ by the fundamental test), the probability that $H_{0}$ be rejected at level $\alpha_{0}$ is

$$
\begin{aligned}
\mathrm{P}\left[\hat{p}_{N}\left(S_{0}\right) \leq \alpha_{0} \mid S_{0}\right] & =\mathrm{P}\left[N \hat{G}_{N}\left(S_{0}\right) \leq(N+1) \alpha_{0}-1 \mid S_{0}\right] \\
& =\mathrm{P}\left[\operatorname{Bi}\left(N, \hat{\alpha}_{0}\right) \leq(N+1) \alpha_{0}-1 \mid S_{0}\right]
\end{aligned}
$$




$$
\begin{aligned}
& \leq \mathrm{P}\left[\operatorname{Bi}(N, \alpha) \leq(N+1) \alpha_{0}-1\right] \\
& =\mathrm{P}\left[\frac{\operatorname{Bi}(N, \alpha)-N \alpha}{(N \alpha(1-\alpha))^{1 / 2}} \leq \frac{N\left(\alpha_{0}-\alpha\right)-\left(1-\alpha_{0}\right)}{(N \alpha(1-\alpha))^{1 / 2}}\right]
\end{aligned}
$$

where the inequality follows on observing that $\hat{\alpha}_{0}>\alpha$ and $\operatorname{Bi}(N, p)$ denotes a binomial random variable with number of trials $N$ and probability of success $p$. From (3.8), we can bound the probability that a Monte Carlo $p$-value as low as $\alpha_{0}$ be obtained when the fundamental test is not significant at level $\alpha$. In particular, for $\alpha_{0}<\alpha$, this probability decreases as the difference $\left|\alpha_{0}-\alpha\right|$ and $N$ get larger. It is also interesting to observe that

$$
\lim _{N \rightarrow \infty} \mathrm{P}\left[\hat{p}_{N}\left(S_{0}\right) \leq \alpha_{0} \mid S_{0}\right]=\lim _{N \rightarrow \infty} \mathrm{P}\left[\frac{\operatorname{Bi}(N, \alpha)-N \alpha}{(N \alpha(1-\alpha))^{1 / 2}} \leq \frac{N\left(\alpha_{0}-\alpha\right)-\left(1-\alpha_{0}\right)}{(N \alpha(1-\alpha))^{1 / 2}}\right]=0
$$

for $\alpha_{0}<\alpha$, so that the probability of a discrepancy between the fundamental test and the Monte Carlo test goes to zero as $N$ increases.

Similarly, for $\hat{\alpha}_{0}<\alpha$ (in which case $H_{0}$ is rejected at level $\alpha$ by the fundamental test), the probability that $H_{0}$ not be rejected at level $\alpha_{0}$ is

$$
\begin{aligned}
\mathrm{P}\left[\hat{p}_{N}\left(S_{0}\right)>\alpha_{0} \mid S_{0}\right] & =\mathrm{P}\left[\operatorname{Bi}\left(N, \hat{\alpha}_{0}\right)>(N+1) \alpha_{0}-1 \mid S_{0}\right] \\
& \leq \mathrm{P}\left[\frac{\operatorname{Bi}(N, \alpha)-N \alpha}{(N \alpha(1-\alpha))^{1 / 2}} \leq \frac{N\left(\alpha_{0}-\alpha\right)-\left(1-\alpha_{0}\right)}{(N \alpha(1-\alpha))^{1 / 2}}\right]
\end{aligned}
$$

hence

$$
\lim _{N \rightarrow \infty} \mathrm{P}\left[\hat{p}_{N}\left(S_{0}\right) \geq \alpha_{0} \mid S_{0}\right]=0, \quad \text { for } \alpha_{0}>\alpha .
$$

(3.10) gives an upper bound on the probability of observing a $p$-value as high as $\alpha_{0}$ when the fundamental test is significant at a level lower than $\alpha$. Again, the probability of a discrepancy between the fundamental test and the Monte Carlo test goes to zero as $N$ increases. The only case where the probability of a discrepancy between the two tests does not go to zero as $N \rightarrow \infty$ is when $\hat{\alpha}_{0}=\alpha$ (an event with probability zero for statistics with continuous distributions).

The probabilities (3.8) and (3.10) may be computed a posteriori to assess the probability of obtaining $p$-values as low (or as high) as $\hat{p}_{N}\left(S_{0}\right)$ when the result of the corresponding fundamental test is actually not significant (or significant) at level $\alpha$. Note also that similar (although somewhat different) calculations may be used to determine the number $N$ of simulations that will ensure a given probability of concordance between the fundamental and the Monte Carlo test [see Marriott (1979)].

\section{Monte Carlo tests with nuisance parameters}

We will now study the case where the distribution of the test statistic $S$ depends on nuisance parameters. We consider a family of probability spaces $\left\{\left(\mathcal{Z}, \mathcal{A}_{\mathcal{Z}}, \mathrm{P}_{\theta}\right): \theta \in \Omega\right\}$ and suppose that $S$ is 
a real valued $\mathcal{A}_{\mathcal{Z}}$-measurable function whose distribution is determined by $\mathrm{P}_{\theta_{0}}$ [i.e., $\theta_{0}$ is the "true" parameter vector]. We wish to test the hypothesis

$$
H_{0}: \theta_{0} \in \Omega_{0}
$$

where $\Omega_{0}$ is a nonempty subset of $\Omega$. Again we take a critical region of the form $S \geq c$, where $c$ is a constant which does not depend on $\theta$. The critical region $S \geq c$ has level $\alpha$ if and only if

$$
\mathrm{P}_{\theta}[S \geq c] \leq \alpha, \forall \theta \in \Omega_{0},
$$

or equivalently,

$$
\sup _{\theta \in \Omega_{0}} \mathrm{P}_{\theta}[S \geq c] \leq \alpha .
$$

Furthermore, $S \geq c$ has size $\alpha$ when

$$
\sup _{\theta \in \Omega_{0}} \mathrm{P}_{\theta}[S \geq c]=\alpha .
$$

If we define the distribution and $p$-value functions,

$$
\begin{aligned}
& F[x \mid \theta]=\mathrm{P}_{\theta}[S \leq x], x \in \overline{\mathbb{R}}, \\
& G[x \mid \theta]=\mathrm{P}_{\theta}[S \geq x], x \in \overline{\mathbb{R}},
\end{aligned}
$$

where $\theta \in \Omega$, it is again easy to see that the critical regions

$$
\sup _{\theta \in \Omega_{0}} G[S \mid \theta] \leq \alpha,
$$

where $\alpha \equiv \sup _{\theta \in \Omega_{0}} G[c \mid \theta]$, and

$$
S \geq \sup _{\theta \in \Omega_{0}} F^{-1}\left[(1-G[c \mid \theta])^{+} \mid \theta\right] \equiv \bar{c}
$$

are equivalent to $S \geq c$ in the sense that $c \leq \bar{c}$, with equality holding when $F[x \mid \theta]$ is discontinuous at $x=c$ for all $\theta \in \Omega_{0}$ or both $F[x \mid \theta]$ and $F^{-1}[q \mid \theta]$ are continuous at $x=c$ and $q=F(c)$ respectively for all $\theta \in \Omega_{0}$, and

$$
\sup _{\theta \in \Omega_{0}} \mathrm{P}_{\theta}[S \geq \bar{c}] \leq \sup _{\theta \in \Omega_{0}} \mathrm{P}_{\theta}[S \geq c]=\sup _{\theta \in \Omega_{0}} \mathrm{P}_{\theta}\left[\sup \left\{G\left[S \mid \theta_{0}\right]: \theta_{0} \in \Omega_{0}\right\} \leq \alpha\right] .
$$

We shall now extend Proposition 2.2 in order to allow for the presence of nuisance parameters. For that purpose, we consider a real random variable $S_{0}$ and random vectors of the form

$$
S(N, \theta)=\left(S_{1}(\theta), \ldots, S_{N}(\theta)\right)^{\prime}, \theta \in \Omega
$$


all defined on a common probability space $\left(\mathcal{Z}, \mathcal{A}_{\mathcal{Z}}, \mathrm{P}\right)$, such that

$$
\begin{aligned}
& \text { the variables } S_{0}, S_{1}\left(\theta_{0}\right), \ldots, S_{N}\left(\theta_{0}\right) \text { are exchangeable for some } \theta_{0} \in \Omega, \\
& \text { each one with distribution function } F\left[x \mid \theta_{0}\right] .
\end{aligned}
$$

Typically, $S_{0}$ will refer to a test statistic computed from observed data when the true parameter vector is $\theta_{0}$ (i.e., $\theta=\theta_{0}$ ), while $S_{1}(\theta), \ldots, S_{N}(\theta)$ will refer to independent and identically distributed (i.i.d.) replications of the test statistic obtained independently (e.g., by simulation) under the assumption that the parameter vector is $\theta$ (i.e., $\mathrm{P}\left[S_{i}(\theta) \leq x\right]=F[x \mid \theta]$ ).

Note that the basic probability measure $\mathrm{P}$ can be interpreted as $\mathrm{P}_{\theta_{0}}$, while the dependence of the distribution of the simulated statistics upon other values of the parameter $\theta$ is expressed by making $S_{i}(\theta)$ a function of $\theta$ (as well as $\omega \in \mathcal{Z}$ ). In parametric models, the statistic $S$ will usually be simulated by first generating an "observation" vector $y$ according to an equation of the form

$$
y=g(\theta, u)
$$

where $u$ has a known distribution (which can be simulated) and then computing

$$
S(\theta) \equiv S[g(\theta, u)] \equiv g_{S}(\theta, u) .
$$

In such cases, the above assumptions can be interpreted as follows: $S_{0}=S\left[y\left(\theta_{0}, u_{0}\right)\right]$ and $S_{i}(\theta)=S\left[y\left(\theta, u_{i}\right)\right], i=1, \ldots, N$, where the random vectors $u_{0}, u_{1}, \ldots, u_{N}$ are i.i.d. (or exchangeable). Note $\theta$ may include the parameters of a disturbance distribution in a model, such as covariance coefficients (or even its complete distribution function), so that the assumption $u$ has a known distribution is not restrictive. Assumptions on the structure of the parameter space $\Omega$ (e.g., whether it is finite-dimensional) will however entail real restrictions on the data-generating process. More generally, it is always possible to consider that the variables $S_{0}, S_{1}(\theta), \ldots, S_{N}(\theta)$ are Pmeasurable by considering their representation in terms of uniform random variables [see Shorack and Wellner (1986, Chapter 1, Theorem 1)]: $S_{0}=F^{-1}\left[V_{0} \mid \theta_{0}\right]$ and $S_{i}(\theta)=F^{-1}\left[V_{i} \mid \theta\right]$, $i=1, \ldots, N$, where $V_{0}, V_{1}, \ldots, V_{N}$ are P-exchangeable with uniform marginal distributions $\left[V_{i} \sim U(0,1), i=0,1, \ldots, N\right]$.

A more general setup that allows for nonparametric models would consist in assuming that the null distribution of the test statistic depends on $\theta$ only through some transformation $T(y)$ of the observation vector $y$, which in turn only depends upon $\theta$ through some transformation $\theta_{*}=h(\theta)$, e.g. a subvector of $\theta$ :

$$
T(y)=g[h(\theta), u]=g\left[\theta_{*}, u\right], \theta_{*} \in \Omega_{*}
$$

where $\Omega_{*}=h(\Omega)$, hence

$$
S(\theta)=S(T(y))=S(g[h(\theta), u]) \equiv g_{S}[h(\theta), u]=g_{S}\left(\theta_{*}, u\right) .
$$

The setup (4.14) - (4.15) allows for reductions of the nuisance parameter space (e.g., through invariance). In particular, nonparametric models may be considered by taking appropriate distributionfree statistics (e.g., test statistics based on signs, ranks, permutations, etc.). What matters for our 
purpose is the possibility of simulating the test statistic, not necessarily the data themselves.

Let also

$$
\begin{gathered}
\hat{F}_{N}[x \mid \theta] \equiv \hat{F}_{N}[x ; S(N, \theta)], \hat{G}_{N}[x \mid \theta] \equiv \hat{G}_{N}[x ; S(N, \theta)] \\
\hat{p}_{N}[x \mid \theta]=\frac{N \hat{G}_{N}[x \mid \theta]+1}{N+1}
\end{gathered}
$$

be defined as in (2.8) - (2.10), and suppose the variables

$$
\begin{gathered}
\sup \left\{\hat{G}_{N}\left[S_{0} \mid \theta\right]: \theta \in \Omega_{0}\right\} \text { and } \inf \left\{\hat{F}_{N}\left[S_{0} \mid \theta\right]: \theta \in \Omega_{0}\right\} \text { are } \mathcal{A}_{\mathcal{Z}} \text {-measurable } \\
\text { where } \Omega_{0} \text { is nonempty subset of } \Omega .
\end{gathered}
$$

For general discussions of measurability of conditions for extrema of random functions, the reader may consult Debreu (1967), Brown and Purves (1973) and Stinchcombe and White (1992). ${ }^{3}$ We then get the following proposition.

Proposition 4.1 VAlidity of MMC tests When ties haVe Zero probability. Under the assumptions and notations (4.10), (4.11) and (4.16) - (4.18), set $S_{0}\left(\theta_{0}\right)=S_{0}$ and suppose that

$$
\mathrm{P}\left[S_{i}\left(\theta_{0}\right)=S_{j}\left(\theta_{0}\right)\right]=0 \quad \text { for } i \neq j, i, j=0,1, \ldots, N .
$$

If $\theta_{0} \in \Omega_{0}$, then for $0 \leq \alpha_{1} \leq 1$,

$$
\begin{aligned}
\mathrm{P}\left[\sup \left\{\hat{G}_{N}\left[S_{0} \mid \theta\right]: \theta \in \Omega_{0}\right\} \leq \alpha_{1}\right] & \leq \mathrm{P}\left[\inf \left\{\hat{F}_{N}\left[S_{0} \mid \theta\right]: \theta \in \Omega_{0}\right\} \geq 1-\alpha_{1}\right] \\
& \leq \frac{I\left[\alpha_{1} N\right]+1}{N+1}
\end{aligned}
$$

where $\mathrm{P}\left[\inf \left\{\hat{F}_{N}\left[S_{0} \mid \theta\right]: \theta \in \Omega_{0}\right\} \geq 1-\alpha_{1}\right]=\mathrm{P}\left[S_{0} \geq \sup \left\{\hat{F}_{N}^{-1}\left[1-\alpha_{1} \mid \theta\right]: \theta \in \Omega_{0}\right\}\right]$ for $0<\alpha_{1}<1$, and

$$
\mathrm{P}\left[\sup \left\{\hat{p}_{N}\left[S_{0} \mid \theta\right]: \theta \in \Omega_{0}\right\} \leq \alpha\right] \leq \frac{I[\alpha(N+1)]}{N+1}, \quad \text { for } 0 \leq \alpha \leq 1 .
$$

Following the latter proposition, if we choose $\alpha_{1}$ and $N$ so that (2.20) holds, the critical region $\sup \left\{\hat{G}_{N}\left[S_{0} \mid \theta\right]: \theta \in \Omega_{0}\right\} \leq \alpha_{1}$ has level $\alpha$ irrespective of the presence of nuisance parameters in the distribution of the test statistic $S$ under the null hypothesis $H_{0}: \theta_{0} \in \Omega_{0}$. The same also holds if we use the (almost) equivalent critical regions $\inf \left\{\hat{F}_{N}\left[S_{0} \mid \theta\right]: \theta \in \Omega_{0}\right\} \geq 1-\alpha_{1}$ or $S_{0} \geq \sup \left\{\hat{F}_{N}^{-1}\left[1-\alpha_{1} \mid \theta\right]: \theta \in \Omega_{0}\right\}$. We shall call such tests maximized Monte Carlo (MMC) tests.

To be more explicit, if $S(\theta)$ is generated according to expressions of the form (4.14) - (4.15),

\footnotetext{
${ }^{3}$ If measurability is an issue, notions of "near-mesurability" can be substituted [see Stinchcombe and White (1992)]. From the viewpoint of getting upper bounds on probabilities, the probability operator can also be replaced by the associated outer measure which is always well-defined [see Dufour (1989, footnote 5)].
} 
we have

$$
\begin{aligned}
\hat{G}_{N}\left[S_{0} \mid \theta\right] & =\frac{1}{N} \sum_{i=1}^{N} \mathbf{1}\left[S_{i}(\theta) \geq S_{0}\right]=\frac{1}{N} \sum_{i=1}^{N} \mathbf{1}\left[S\left(g\left[h(\theta), u_{i}\right]\right) \geq S_{0}\right] \\
& =\frac{1}{N} \sum_{i=1}^{N} \mathbf{1}\left[g_{S}\left(\theta_{*}, u_{i}\right) \geq S_{0}\right] .
\end{aligned}
$$

The function $\hat{G}_{N}\left[S_{0} \mid \theta\right]$ (or $\hat{p}_{N}\left[S_{0} \mid \theta\right]$ ), is then maximized with respect to $\theta \in \Omega_{0}$ [or equivalently, with respect to $\theta_{*} \in \Omega_{0 *}=h\left(\Omega_{0}\right)$ ] keeping the observed statistic $S_{0}$ and the simulated disturbance vectors $u_{1}, \ldots, u_{N}$ fixed. The function $\hat{G}_{N}\left[S_{0} \mid \theta\right]$ is a step-type function which typically has zero derivatives almost everywhere, except on isolated points (or manifolds) where it is not differentiable. Further, the supremum of $\hat{G}_{N}\left[S_{0} \mid \theta\right]$ is typically not unique, in the sense that several values of $\theta$ will yield the required supremum. So it cannot be maximized with usual derivative-based algorithms. However, the required maximizations can be performed by using appropriate optimization algorithms that do not require differentiability, such as simulated annealing. For further discussion of such algorithms, the reader may consult Goffe, Ferrier and Rogers (1994).

It is easy to extend Proposition $\mathbf{4 . 1}$ in order to relax the no-tie assumption (4.19). For that purpose, we generate as in Proposition $\mathbf{2 . 4}$ a vector $\left(U_{0}, U_{1}, \ldots, U_{N}\right)$ of $N+1$ i.i.d. $U(0,1)$ random variables independent of $S_{0}, S_{1}\left(\theta_{0}\right), \ldots S_{N}\left(\theta_{0}\right)$, and we consider the corresponding randomized distribution, tail area and $p$-value functions:

$$
\begin{gathered}
\tilde{F}_{N}[x \mid \theta] \equiv \tilde{F}_{N}\left[x ; U_{0}, S(N, \theta), U(N)\right] \\
\tilde{G}_{N}[x \mid \theta] \equiv \tilde{G}_{N}\left[x ; U_{0}, S(N, \theta), U(N)\right], \tilde{p}_{N}[x \mid \theta]=\frac{N \tilde{G}_{N}[x \mid \theta]+1}{N+1}
\end{gathered}
$$

where

$$
U(N)=\left(U_{1}, \ldots, U_{N}\right) \text {, and } U_{0}, U_{1}, \ldots, U_{N} \stackrel{i . i . d .}{\sim} U(0,1) .
$$

Under the corresponding measurability assumption

$$
\begin{gathered}
\sup \left\{\tilde{G}_{N}\left[S_{0} \mid \theta\right]: \theta \in \Omega_{0}\right\} \text { and } \inf \left\{\tilde{F}_{N}\left[S_{0} \mid \theta\right]: \theta \in \Omega_{0}\right\} \text { are } \mathcal{A}_{\mathcal{Z}} \text {-measurable } \\
\text { where } \Omega_{0} \text { is nonempty subset of } \Omega,
\end{gathered}
$$

we can then state the following generalization of Proposition $\mathbf{2 . 4}$.

Proposition 4.2 VALIDITy OF MMC tests FOR GENERAL STATISTICS. Under the assumptions and notations (4.10), (4.11), (4.16) - (4.18) and (4.23) - (4.26), suppose $U_{0}, U_{1}, \ldots, U_{N}$ are independent of $S_{0}, S_{1}\left(\theta_{0}\right), \ldots, S_{N}\left(\theta_{0}\right)$. If $\theta_{0} \in \Omega_{0}$, then for $0 \leq \alpha_{1} \leq 1$ and for $0 \leq \alpha \leq 1$,

$$
\mathrm{P}\left[\sup \left\{\hat{G}_{N}\left[S_{0} \mid \theta\right]: \theta \in \Omega_{0}\right\} \leq \alpha_{1}\right] \leq \mathrm{P}\left[\sup \left\{\tilde{G}_{N}\left[S_{0} \mid \theta\right]: \theta \in \Omega_{0}\right\} \leq \alpha_{1}\right] \leq \frac{I\left[\alpha_{1} N\right]+1}{N+1},
$$




$$
\begin{gathered}
\mathrm{P}\left[\sup \left\{\hat{G}_{N}\left[S_{0} \mid \theta\right]: \theta \in \Omega_{0}\right\} \leq \alpha_{1}\right] \leq \mathrm{P}\left[\sup \left\{\tilde{F}_{N}\left[S_{0} \mid \theta\right]: \theta \in \Omega_{0}\right\} \geq 1-\alpha_{1}\right] \leq \frac{I\left[\alpha_{1} N\right]+1}{N+1}, \\
\mathrm{P}\left[\sup \left\{\hat{p}_{N}\left[S_{0} \mid \theta\right]: \theta \in \Omega_{0}\right\} \leq \alpha\right] \leq \mathrm{P}\left[\sup \left\{\tilde{p}_{N}\left[S_{0} \mid \theta\right]: \theta \in \Omega_{0}\right\} \leq \alpha\right] \leq \frac{I[\alpha(N+1)]}{N+1} .
\end{gathered}
$$

One should note here that the validity results of propositions 4.1 and 4.2 differ from those of the corresponding propositions $\mathbf{2 . 2}$ and $\mathbf{2 . 4}$ in the sense that equalities have been replaced by inequalities. This entails that the corresponding maximized $\mathrm{MC}$ tests is exact in the sense that the probability of type I error cannot be larger than the nominal level $\alpha$ of the test, but its size may be lower that the level (leading to a conservative procedure). ${ }^{4}$ In view of the fact the distribution of the test statistic involves nuisance parameters, this is not surprising: since the distribution of the test statistic varies as a function of nuisance parameters, we can expect that the probability of type I error be lower than the level $\alpha$ for some distribution compatible with the null hypothesis, even if we use the tightest possible critical value that allows one to control the level of the test. Both the fundamental (infeasible) test and its MC version are not similar. This is a feature of the test statistic, not its $\mathrm{MC}$ implementation. Of course, it is preferable from the power viewpoint that the discrepancy between the size of the test and its level be as small as possible. This discrepancy would disappear if we could estimate and maximize without error the theoretical $p$-value function $G[x \mid \theta]$ or the appropriate critical value, but this is not typically feasible. In general, the discrepancy between the size and the nominal level of the test depends on the form of the test statistic, the null hypothesis, and the number $N$ of replications of the MMC procedure. Studying in any detail this sort of effect would go beyond the scope of the present. ${ }^{5}$

\section{Asymptotic Monte Carlo tests based on a consistent set estimator}

In this section, we propose simplified approximate versions of the procedures proposed in the previous section when a consistent point or set estimate of $\theta$ is available. To do this, we shall need to reformulate the setup used previously in order to allow for an increasing sample size.

Consider

$$
S_{T 0}, S_{T 1}(\theta), \ldots, S_{T N}(\theta), \quad T \geq I_{0}, \theta \in \Omega,
$$

real random variables all defined on a common probability space $\left(\mathcal{Z}, \mathcal{A}_{\mathcal{Z}}, \mathrm{P}\right)$, and set

$$
S_{T}(N, \theta)=\left(S_{T 1}(\theta), \ldots, S_{T N}(\theta)\right), \quad T \geq I_{0} .
$$

We will be primarily interested by situations where

$$
\text { the variables } S_{T 0}, S_{T 1}\left(\theta_{0}\right), \ldots, S_{T N}\left(\theta_{0}\right) \text { are exchangeable for some } \theta_{0} \in \Omega,
$$

\footnotetext{
${ }^{4}$ We say that a test procedure is conservative at level $\alpha$ if its size is strictly smaller than $\alpha$. Note that a non-similar test is not conservative as long as its size is equal to the level $\alpha$ (even though the probability of type I error is smaller than $\alpha$ for certain distributions compatible with the null hypothesis).

${ }^{5} \mathrm{~A}$ question of interest here consists in studying the conditions under which the discrepancy will disappear as the number of MC replications goes to infinity $(N \rightarrow \infty)$. The reader will also find simulation evidence on the size and power properties of MMC procedures in Dufour and Khalaf (2003a, 2003b) Dufour and Jouini (2004) and Dufour and Valéry (2004).
} 
each one with distribution function $F_{T}\left[x \mid \theta_{0}\right]$.

Here $S_{T 0}$ will normally refer to a test statistic with distribution function $F_{T}[\cdot \mid \theta]$ based on a sample of size $T$, while $S_{T 1}(\theta), \ldots, S_{T N}(\theta)$ are i.i.d. replications of the same test statistic obtained independently under the assumption that the parameter vector is $\theta$ (i.e., $\mathrm{P}\left[S_{T i}(\theta) \leq x\right]=$ $\left.F_{T}[x \mid \theta], i=1, \ldots, N\right)$. Let also

$$
\begin{gathered}
\hat{F}_{T N}[x \mid \theta]=\hat{F}_{N}\left[x ; S_{T}(N, \theta)\right], \hat{G}_{T N}[x \mid \theta]=\hat{G}_{N}\left[x ; S_{T}(N, \theta)\right], \\
\hat{p}_{T N}[x \mid \theta]=\frac{N \hat{G}_{T N}[x \mid \theta]+1}{N+1},
\end{gathered}
$$

and let $\hat{F}_{T N}^{-1}[x \mid \theta]$ be defined as in (2.7) - (2.10).

We consider first the situation where $p$-values are maximized over a subset $C_{T}$ of $\Omega$ (e.g., a confidence set for $\theta$ ) instead of $\Omega_{0}$. Consequently, we introduce the following assumption:

$C_{T}, T \geq I_{0}$ is a sequence of (possibly random) subsets of $\Omega$ such that $\sup \left\{\hat{G}_{T N}\left[S_{T 0} \mid \theta\right]: \theta \in C_{T}\right\}$ and $\inf \left\{\hat{F}_{T N}\left[S_{T 0} \mid \theta\right]: \theta \in C_{T}\right\}$ are $\mathcal{A}_{\mathcal{Z}}$-measurable, for all $T \geq I_{0}$, where $\Omega_{0}$ is nonempty subset of $\Omega$.

Then we have the following proposition.

Proposition 5.1 ASYMPTOTIC VALIDITY OF CONFIDENCE-SET RESTRICTED MMC TESTS: CONTINUOUS DISTRIBUTIONS. Under the assumptions and notations (5.1) to (5.6), set $S_{T 0}\left(\theta_{0}\right)=S_{T 0}$, suppose

$$
\mathrm{P}\left[S_{T i}\left(\theta_{0}\right)=S_{T j}\left(\theta_{0}\right)\right]=0 \text { for } i \neq j \text {, and } i, j=0,1, \ldots, N,
$$

for all $T \geq I_{0}$, and let $C_{T}, T \geq I_{0}$, be a sequence of (possibly random) subsets of $\Omega$ such that

$$
\lim _{T \rightarrow \infty} \mathrm{P}\left[\theta_{0} \in C_{T}\right]=1 .
$$

If $\theta_{0} \in \Omega_{0}$, then

$$
\begin{aligned}
\lim _{T \rightarrow \infty} \mathrm{P}\left[\sup \left\{\hat{G}_{T N}\left[S_{T 0} \mid \theta\right]: \theta \in C_{T}\right\} \leq \alpha_{1}\right] \\
\quad \leq \lim _{T \rightarrow \infty} \mathrm{P}\left[\inf \left\{\hat{F}_{T N}\left[S_{T 0} \mid \theta\right]: \theta \in C_{T}\right\} \geq 1-\alpha_{1}\right] \\
\quad=\lim _{T \rightarrow \infty} \mathrm{P}\left[S_{T 0} \geq \sup \left\{\hat{F}_{T N}^{-1}\left[1-\alpha_{1} \mid \theta\right]: \theta \in C_{T}\right\}\right] \leq \frac{I\left[\alpha_{1} N\right]+1}{N+1}
\end{aligned}
$$

and

$$
\lim _{T \rightarrow \infty} \mathrm{P}\left[\sup \left\{\hat{p}_{T N}\left[S_{T 0} \mid \theta\right]: \theta \in C_{T}\right\} \leq \alpha\right] \leq \frac{I[\alpha(N+1)]}{N+1}, \text { for } 0 \leq \alpha \leq 1 .
$$


It is quite easy to find a consistent set estimate of $\theta_{0}$ whenever a consistent point estimate $\hat{\theta}_{T}$ of $\theta_{0}$ is available. Suppose $\Omega \subseteq \mathbb{R}^{k}$ and

$$
\lim _{T \rightarrow \infty} \mathrm{P}\left[\left\|\hat{\theta}_{T}-\theta_{0}\right\|<\varepsilon\right]=1, \forall \varepsilon>0
$$

where $\|\cdot\|$ is the Euclidean norm in $\mathbb{R}^{k}$ [i.e., $\|x\|=\left(x^{\prime} x\right)^{1 / 2}, x \in \mathbb{R}^{k}$ ]. Note that condition (5.8) need only hold for the true value $\theta_{0}$ of the parameter vector $\theta$. Then any set of the form $C_{T}=\left\{\theta \in \Omega:\left\|\hat{\theta}_{T}-\theta\right\|<c\right\}$ satisfies (5.8), whenever $c$ is a fixed positive constant that does not depend on $T$. More generally, if there is a sequence of (possibly random) matrices $A_{T}$ and a non-negative exponent $\delta$ such that

$$
\lim _{T \rightarrow \infty} \mathrm{P}\left[T^{\delta}\left\|A_{T}\left(\hat{\theta}_{T}-\theta_{0}\right)\right\|^{2}<c\right]=1, \forall c>0,
$$

then any set of the form

$$
\begin{aligned}
C_{T} & =\left\{\theta \in \Omega:\left(\hat{\theta}_{T}-\theta\right)^{\prime} A_{T}^{\prime} A_{T}\left(\hat{\theta}_{T}-\theta\right)<c / T^{\delta}\right\} \\
& =\left\{\theta \in \Omega:\left\|A_{T}\left(\hat{\theta}_{T}-\theta\right)\right\|^{2}<c / T^{\delta}\right\}, c>0
\end{aligned}
$$

satisfies (5.8), since in this case,

$$
\begin{aligned}
\mathrm{P}\left[\theta_{0} \in C_{T}\right] & =\mathrm{P}\left[\left(\hat{\theta}_{T}-\theta_{0}\right)^{\prime} A_{T}^{\prime} A_{T}\left(\hat{\theta}_{T}-\theta_{0}\right)<c / T^{\delta}\right] \\
& =\mathrm{P}\left[T^{\delta}\left(\hat{\theta}_{T}-\theta_{0}\right)^{\prime} A_{T}^{\prime} A_{T}\left(\hat{\theta}_{T}-\theta_{0}\right)<c\right] \underset{T \rightarrow \infty}{\longrightarrow} 1 .
\end{aligned}
$$

In particular (5.12) will hold whenever we can find $\bar{\delta}>0($ e.g., $\bar{\delta}=1)$ such that $T^{\bar{\delta} / 2} A_{T}\left(\hat{\theta}_{T}-\theta_{0}\right)$ has an asymptotic distribution as $T \rightarrow \infty$ and $\delta$ is selected so that $0 \leq \delta<\bar{\delta}$. Whenever $\delta>0$ and $\underset{T \rightarrow \infty}{\operatorname{plim}}\left(A_{T}^{\prime} A_{T}\right)=C_{0}$ with $\operatorname{det}\left(C_{0}\right) \neq 0$, the diameter of the set $C_{T}$ goes to zero, a fact which can greatly simplifies the evaluation of the variables $\sup \left\{\hat{G}_{T N}\right\}, \inf \left\{\hat{F}_{T N}\right\}$ and $\sup \left\{\hat{p}_{T N}\right\}$ in (5.9) - (5.10).

The above procedure may be especially useful when the distribution of the test statistic is highly sensitive to nuisance parameters, in a way that would make its asymptotic distribution discontinuous with respect to the nuisance parameters. In such cases, a simulation-based procedure where the nuisance parameters are replaced by a consistent point estimate - such as a parametric bootstrap procedure - may not converge to the appropriate asymptotic distribution (because the point estimate does not converge sufficiently fast to overcome the discontinuity). Here, possible discontinuities in the asymptotic distribution are automatically taken into account thorough a numerical maximization over a set that contains the correct value of the nuisance parameter with a probability asymptotically equal to one: using a consistent set estimator as opposed a point estimate (which does not converge fast enough) can overcome such a high sensitivity to nuisance parameters. Of course, the procedure can also be helpful in situations where the finite-sample distribution is highly sensitive to nuisance parameters, even though it does not lead to asymptotic failure of the bootstrap.

Again, it is possible to extend Proposition 5.1 to statistics with general (possibly discrete) dis- 
tributions by considering properly randomized distribution, tail area and $p$-value functions:

$$
\begin{aligned}
\tilde{F}_{T N}[x \mid \theta] & =\tilde{F}_{N}\left[x ; U_{0}, S_{T}(N ; \theta), U(N)\right], \\
\tilde{G}_{T N}[x \mid \theta] & =\tilde{G}_{N}\left[x ; U_{0}, S_{T}(N ; \theta), U(N)\right], \\
\tilde{p}_{T N}[x \mid \theta] & =\frac{N \tilde{G}_{T N}[x \mid \theta]+1}{N+1},
\end{aligned}
$$

where $\tilde{F}_{N}[\cdot], \tilde{G}_{N}[\cdot], U_{0}$ and $U(N)$ are defined as in (4.23) - (4.25).

Proposition 5.2 ASYMPTOTIC VALIDITY OF CONFIDENCE-SET RESTRICTED MMC TESTS: GENERAL DISTRIBUTIONS. Under the assumptions and notations (5.1) - (5.6) and (5.14) - (5.16), suppose the sets $C_{T} \subseteq \Omega, T \geq I_{0}$, satisfy (5.8). If $\theta_{0} \in \Omega_{0}$, then for $0 \leq \alpha_{1} \leq 1$ and $0 \leq \alpha \leq 1$,

$$
\begin{aligned}
\lim _{T \rightarrow \infty} \mathrm{P}\left[\sup \left\{\hat{G}_{T N}\left[S_{T 0} \mid \theta\right]: \theta \in C_{T}\right\} \leq \alpha_{1}\right] & \leq \lim _{T \rightarrow \infty} \mathrm{P}\left[\sup \left\{\tilde{G}_{T N}\left[S_{T 0} \mid \theta\right]: \theta \in C_{T}\right\} \leq \alpha_{1}\right] \\
& \leq \frac{I\left[\alpha_{1} N\right]+1}{N+1} \\
\lim _{T \rightarrow \infty} \mathrm{P}\left[\sup \left\{\hat{G}_{T N}\left[S_{T 0} \mid \theta\right]: \theta \in C_{T}\right\} \leq \alpha_{1}\right] & \leq \lim _{T \rightarrow \infty} \mathrm{P}\left[\sup \left\{\tilde{F}_{T N}\left[S_{T 0} \mid \theta\right]: \theta \in C_{T}\right\} \geq 1-\alpha_{1}\right] \\
& \leq \frac{I\left[\alpha_{1} N\right]+1}{N+1} \\
& \leq \lim _{T \rightarrow \infty} \mathrm{P}\left[\sup \left\{\tilde{p}_{T N}\left[S_{0} \mid \theta\right]: \theta \in C_{T}\right\} \leq \alpha\right] \\
\lim _{T \rightarrow \infty} \mathrm{P}\left[\sup \left\{\hat{p}_{T N}\left[S_{T 0} \mid \theta\right]: \theta \in C_{T}\right\} \leq \alpha\right] & \leq \frac{I[\alpha(N+1)]}{N+1} .
\end{aligned}
$$

\section{Asymptotic Monte Carlo tests based on consistent point estimate}

Parametric bootstrap tests may be interpreted as a simplified form of the procedures described in Proposition $\mathbf{5 . 1}$ and $\mathbf{5 . 2}$ where the consistent confidence set $C_{T}$ has been replaced by a consistent point estimate $\hat{\theta}_{T}$. In other words, the distribution of $S_{T}(\theta), \theta \in \Omega_{0}$, is simulated at a single point $\hat{\theta}_{T}$, leading to a local (or pointwise) MC test. It is well known that such bootstrap tests are not generally valid, unless stronger regularity conditions are imposed. In the following proposition, we extend earlier proofs of the asymptotic validity of such bootstrap tests. In particular, we allow for the presence of nuisance parameters in the asymptotic distribution of the test statistic considered. Further, our proofs have the interesting feature of being cast in the MC test setup where the number of replications $N$ is kept fixed even asymptotically.

Such pointwise procedures require stronger regularity assumptions (such as uniform continuity and convergence over the nuisance parameter space) - so that they may fail in irregular cases where the maximized procedures described in the previous sections succeed in controlling the level of the test (at least asymptotically). But they are simpler to implement and may be taken as a natural 
starting point for implementing maximized procedures. In particular, if a pointwise MC $p$-value is larger than the level $\alpha$ of the test (so that the pointwise MC test is not significant at level $\alpha$ ), it is clear that the maximized $p$-value must be larger than $\alpha$ (so that the maximized MC test is not significant at level $\alpha$ )

In order to establish a clear asymptotic validity result, we will use four basic assumptions on the distributions of the statistics $S_{T}(\theta)$ as functions of the parameter vector $\theta$ :

$$
\begin{gathered}
S_{T 1}(\theta), \ldots, S_{T N}(\theta) \text { are } i . i . d \text {. according to the distribution } \\
F_{T}[x \mid \theta]=\mathrm{P}\left[S_{T}(\theta) \leq x\right], \forall \theta \in \Omega ; \\
\Omega \text { is a nonempty subset of } \mathbb{R}^{k}
\end{gathered}
$$

for every $T \geq I_{0}, S_{T 0}$ is a real random variable and $\hat{\theta}_{T}$ an estimator of $\theta$, both measurable with respect to the probability space $\left(\mathcal{Z}, \mathcal{A}_{\mathcal{Z}}, \mathrm{P}\right)$, and $F_{T}\left[S_{T 0} \mid \hat{\theta}_{T}\right]$ is a random variable;

$$
\begin{aligned}
\forall \varepsilon_{0}>0, \forall \varepsilon_{1}>0, & \exists \delta>0 \text { and a sequence of open subsets } D_{T 0}\left(\varepsilon_{0}\right) \text { in } \mathbb{R} \text { such that } \\
& \liminf _{T \rightarrow \infty} \mathrm{P}\left[S_{T 0} \in D_{T 0}\left(\varepsilon_{0}\right)\right] \geq 1-\varepsilon_{0} \text { and } \\
\left\|\theta-\theta_{0}\right\| \leq \delta \Rightarrow & \limsup _{T \rightarrow \infty}\left\{\sup _{x \in D_{T 0}\left(\varepsilon_{0}\right)}\left|F_{T}[x \mid \theta]-F_{T}\left[x \mid \theta_{0}\right]\right|\right\} \leq \varepsilon_{1} .
\end{aligned}
$$

The first of these four conditions replaces the exchangeability assumption by an assumption of i.i.d. variables. The two next ones simply make appropriate measurability assumptions, while the last one may be interpreted as a local equicontinuity condition $\left(\right.$ at $\left.\theta=\theta_{0}\right)$ on the sequence of distribution functions $F_{T}(x \mid \theta), T \geq I_{0}$. Note that $S_{T 0}$ is not assumed to follow the same distribution as the other variables $S_{T 1}(\theta), \ldots, S_{T N}(\theta)$. Furthermore, $S_{T 0}$ and $F_{T}(x \mid \theta)$ do not necessarily converge to limits as $T \rightarrow \infty$. An alternative assumption of interest would consist in assuming that $S_{T 0}$ converges in probability $(\stackrel{p}{\longrightarrow})$ to a random variable $\bar{S}_{0}$ as $T \rightarrow \infty$, in which case the "global" equicontinuity condition (6.4) can be weakened to a "local" one:

$$
S_{T 0} \underset{T \rightarrow \infty}{\stackrel{p}{\longrightarrow}} \bar{S}_{0}
$$

$D_{0}$ is subset of $\mathbb{R}$ such that $\mathrm{P}\left[\bar{S}_{0} \in D_{0}\right.$ and $S_{T 0} \in D_{0}$ for all $\left.T \geq I_{0}\right]=1$;

$\forall x \in D_{0}, \forall \varepsilon>0, \exists \delta>0$ and an open neighborhood $B(x, \varepsilon)$ of $x$ such that

$$
\left\|\theta-\theta_{0}\right\| \leq \delta \Rightarrow \limsup _{T \rightarrow \infty}\left\{\sup _{y \in B(x, \varepsilon) \cap D_{0}}\left|F_{T}[y \mid \theta]-F_{T}\left[y \mid \theta_{0}\right]\right|\right\} \leq \varepsilon .
$$

We can now show that Monte Carlo tests obtained by simulating $S_{T i}(\theta), i=1, \ldots, N$, with $\theta=\hat{\theta}_{T}$ are equivalent for large $T$ to those based on using the true value $\theta=\theta_{0}$. 
Proposition 6.1 ASYMPTOTIC VALIDITY OF BOOTSTRAP $p$-VALUES. Under the assumptions and notations (5.1), (5.2), (5.4), (5.5), (5.14) - (5.16) and (6.1) - (6.3), suppose the random variable $S_{T 0}$ and the estimator $\hat{\theta}_{T}$ are both independent of $S_{T}(N, \theta)$ and $U_{0}$. If $\operatorname{plim}_{T \rightarrow \infty} \hat{\theta}_{T}=\theta_{0}$ and condition (6.4) or (6.5) - (6.7) hold, then for $0 \leq \alpha_{1} \leq 1$ and $0 \leq \alpha \leq 1$,

$$
\begin{aligned}
& \lim _{T \rightarrow \infty}\left\{\mathrm{P}\left[\tilde{G}_{T N}\left[S_{T 0} \mid \hat{\theta}_{T}\right] \leq \alpha_{1}\right]-\mathrm{P}\left[\tilde{G}_{T N}\left[S_{T 0} \mid \theta_{0}\right] \leq \alpha_{1}\right]\right\} \\
& \quad=\lim _{T \rightarrow \infty}\left\{\mathrm{P}\left[\hat{G}_{T N}\left[S_{T 0} \mid \hat{\theta}_{T}\right] \leq \alpha_{1}\right]-\mathrm{P}\left[\hat{G}_{T N}\left[S_{T 0} \mid \theta_{0}\right] \leq \alpha_{1}\right]\right\}=0,
\end{aligned}
$$

and

$$
\begin{aligned}
& \lim _{T \rightarrow \infty}\left\{\mathrm{P}\left[\tilde{p}_{T N}\left[S_{T 0} \mid \hat{\theta}_{T}\right] \leq \alpha\right]-\mathrm{P}\left[\tilde{p}_{T N}\left[S_{T 0} \mid \theta_{0}\right] \leq \alpha\right]\right\} \\
& \quad=\lim _{T \rightarrow \infty}\left\{\mathrm{P}\left[\hat{p}_{T N}\left[S_{T 0} \mid \hat{\theta}_{T}\right] \leq \alpha\right]-\mathrm{P}\left[\hat{p}_{T N}\left[S_{T 0} \mid \theta_{0}\right] \leq \alpha\right]\right\}=0 .
\end{aligned}
$$

It is worth noting that condition (6.7) holds whenever $F_{T}[x \mid \theta]$ converges to a distribution function $F_{\infty}[x \mid \theta]$ which is continuous with respect to $\left(x, \theta^{\prime}\right)^{\prime}$, for $x \in D_{0}$, as follows:

$$
\begin{aligned}
& \forall x \in D_{0}, \forall \varepsilon>0, \exists \delta_{1}>0 \text { and an open neighborhood } B_{1}(x, \varepsilon) \text { of } x \text { such that } \\
& \left\|\theta-\theta_{0}\right\| \leq \delta \Rightarrow \limsup _{T \rightarrow \infty}\left(\sup _{y \in B_{1}(x, \varepsilon) \cap D_{0}}\left|F_{T}[y \mid \theta]-F_{\infty}[y \mid \theta]\right|\right) \leq \varepsilon \\
& \forall x \in D_{0}, \forall \varepsilon>0, \exists \delta_{2}>0 \text { and an open neighborhood } B_{2}(x, \varepsilon) \text { of } x \text { such that } \\
& \left\|\theta-\theta_{0}\right\| \leq \delta_{2} \Rightarrow \sup _{y \in B_{2}(x, \varepsilon) \cap D_{0}}\left|F_{\infty}[y \mid \theta]-F_{\infty}\left[y \mid \theta_{0}\right]\right| \leq \varepsilon
\end{aligned}
$$

It is then easy to see that (6.10) - (6.11) entail (6.7) on noting that

$$
\begin{aligned}
\left|F_{T}[x \mid \theta]-F_{T}\left[x \mid \theta_{0}\right]\right| \leq & \left|F_{T}[x \mid \theta]-F_{\infty}[x \mid \theta]\right|+\left|F_{T}\left[x \mid \theta_{0}\right]-F_{\infty}\left[x \mid \theta_{0}\right]\right| \\
& +\left|F_{\infty}[x \mid \theta]-F_{\infty}\left[x \mid \theta_{0}\right]\right|, \forall x .
\end{aligned}
$$

Note also that (6.11) holds whenever $F_{\infty}[x \mid \theta]$ is continuous with respect to $\left(x, \theta^{\prime}\right)^{\prime}$, although the latter condition is not at all necessary (e.g., in models where $D_{0}$ is a discrete set of points). In particular, (6.10) - (6.11) will hold when $F_{T}[x \mid \theta]$ admits an expansion around a pivotal distribution:

$$
F_{T}[x \mid \theta]=F_{\infty}(x)+T^{-\gamma} g(x, \theta)+h_{T}(x, \theta)
$$

where $F_{\infty}(x)$ is a distribution function that does not depend on $\theta, \gamma>0$, with the following assumptions on $g(x, \theta)$ and $h_{T}(x, \theta)$ :

$\forall x \in D_{0}, \exists$ an open neighborhood $B\left(x, \theta_{0}\right)$ of $\left(x, \theta_{0}^{\prime}\right)^{\prime}$ such that $|g(y, \theta)| \leq C(x, \theta)$, for all $\left(y, \theta^{\prime}\right)^{\prime} \in B\left(x, \theta_{0}\right) \cap D_{0}$

where $C(x, \theta)$ is a positive constant, and 


$$
T^{\gamma} h_{T}(y, \theta) \underset{T \rightarrow \infty}{\longrightarrow} 0 \text { uniformly on } B\left(x, \theta_{0}\right) \cap D_{0} .
$$

The latter is quite similar (although somewhat weaker) than the assumption considered by Hall and Titterington (1989, eq. (2.5))].

When $S_{T 0}$ is distributed like $S_{T}\left(\theta_{0}\right)$, i.e., $\mathrm{P}\left[S_{T 0} \leq x\right]=F_{T}\left[x \mid \theta_{0}\right]$, we can apply Proposition 2.4 and see that $\mathrm{P}\left[\tilde{G}_{T N}\left[S_{T 0} \mid \theta_{0}\right] \leq \alpha_{1}\right]=\left(I\left[\alpha_{1} N\right]+1\right) /(N+1)$, hence

$$
\lim _{T \rightarrow \infty} \mathrm{P}\left[\tilde{G}_{T N}\left[S_{T 0} \mid \hat{\theta}_{T}\right] \leq \alpha_{1}\right]=\frac{I\left[\alpha_{1} N\right]+1}{N+1} .
$$

\section{Conclusion}

In this paper, we have made four main contributions. First, for the case where we have a test statistic whose distribution does not involve nuisance parameters under the null hypothesis, we have proposed a general form of Monte Carlo testing which allows for exchangeable (as opposed to i.i.d.) Monte Carlo replications of general test statistics whose distribution can take an arbitrary form (continuous, discrete or mixed). In particular, this form is not limited to permutation tests, which has received considerable attention in the earlier literature on Monte Carlo tests [see Dwass (1957), Green (1977), Vadiveloo (1983), Keller-McNulty and Higgins (1987), Lock (1991), Edgington (1995), Manly (1997), Noreen (1989), Good (1994)]. Second, we have shown how the method can be extended to models with nuisance parameters as long as the null distribution of the test statistic can be simulated once the nuisance parameters have been specified. This leads to what we called maximized Monte Carlo tests which were shown to satisfy the level constraint. Thirdly, we proposed a simplified version of the latter method which can lead to asymptotically valid tests even if the asymptotic distribution depends on nuisance parameters in a discontinuous way. This method only requires one to use a consistent set estimator of the nuisance parameters, which is always feasible as long as a consistent point estimate of the nuisance parameters is available. Further, in the latter case, no additional information is required on the asymptotic distribution of the consistent estimator. Fourth, we showed that Monte Carlo tests obtained on replacing unknown nuisance parameters by consistent estimates also lead to asymptotically valid tests. However, it is important to note that stronger conditions are needed for this to occur and such conditions may be difficult to check in practice.

The main shortcoming of the proposed MMC tests comes from the fact that such tests may be computationally demanding. We cannot study here the appropriate numerical algorithms or detailed implementations of the theory described above. But a number of such applications are presented in companion papers (which are based on earlier versions of the present paper). For example, for an illustration of the adjustment for discreteness proposed here, the reader may consult Dufour, Farhat, Gardiol and Khalaf (1998) where it is used to correct the size of Kolmogorov-Smirnov tests (which involve a discrete statistic) for the normality of errors in a linear regression. The method of maximized Monte Carlo tests can of course be applied to a wide array of models where nuisance parameters problems show up: for example, inference in seemingly unrelated regressions [Dufour and Khalaf (2003a)], simultaneous equations models [Dufour and Khalaf (2003b)], dynamic models [Dufour and Jouini (2004), Dufour and Valéry (2004)], and models with limited dependent variables 
[Jouneau-Sion and Torrès (2003)]. It is clear many more applications are possible. The size and power properties of the proposed procedures are also studied by simulation methods in this work. 


\section{A. Appendix: Proofs}

Proof of Lemma 2.1 By condition (2.12), the variables $y_{1}, y_{2}, \ldots, y_{N}$ are all distinct with probability 1 , and the rank vector $\left(R_{1}, R_{2}, \ldots, R_{N}\right)^{\prime}$ is with probability 1 a permutation of the integers $(1,2, \ldots, N)$. Furthermore, since the variables $y_{1}, y_{2}, \ldots, y_{N}$ are exchangeable, the $N$ ! distinct permutations of $\left(y_{1}, y_{2}, \ldots, y_{N}\right)$ have the same probability $1 / N$ !. Consequently, we have:

$$
\begin{gathered}
\mathrm{P}\left(R_{j}=i\right)=\frac{1}{N}, i=1,2, \ldots, N, \\
\mathrm{P}\left(R_{j} / N \leq x\right)=I[x N] / N, 0 \leq x \leq 1,
\end{gathered}
$$

from which (2.13) follows and

$$
\begin{aligned}
\mathrm{P}\left(R_{j} / N<x\right) & =(I[x N]-1) / N, & & \text { if } x N \in \mathbb{Z}_{+}, \\
& =I[x N] / N, & & \text { otherwise, }
\end{aligned}
$$

where $\mathbb{Z}_{+}$is the set of the positive integers. Since, for any real number $z$,

$$
\begin{aligned}
I[N-z] & =N-z, & & \text { if } z \text { is an integer, } \\
& =N-I[z]-1, & & \text { otherwise }
\end{aligned}
$$

we then have, for $0 \leq x \leq 1$,

$$
\begin{aligned}
\mathrm{P}\left(R_{j} / N \geq x\right) & =1-\mathrm{P}\left(R_{N} / N<x\right)=(N-I[x N]+1) / N, & & \text { if } x N \in \mathbb{Z}_{+}, \\
& =(N-I[x N]) / N & & \text { otherwise, }
\end{aligned}
$$

hence

$$
\begin{aligned}
\mathrm{P}\left[R_{j} / N \geq x\right] & =(I[(1-x) N]+1) / N, & & \text { if } 0<x \leq 1, \\
& =1, & & \text { if } x=0,
\end{aligned}
$$

from which we get (2.14).

Proof of Proposition 2.2 Assuming there are no ties among $S_{0}, S_{1}, \ldots, S_{N}$ (an event with probability 1), we have

$$
\begin{aligned}
\hat{G}_{N}\left(S_{0}\right) & =\frac{1}{N} \sum_{i=1}^{N} \mathbf{1}\left(S_{i} \geq S_{0}\right)=\frac{1}{N} \sum_{i=1}^{N}\left[1-\mathbf{1}\left(S_{i} \leq S_{0}\right)\right]=1-\frac{1}{N} \sum_{i=1}^{N} \mathbf{1}\left(S_{i} \leq S_{0}\right) \\
& =1-\frac{1}{N}\left[-1+\sum_{i=0}^{N} \mathbf{1}\left(S_{i} \leq S_{0}\right)\right]=\left(N+1-R_{0}\right) / N
\end{aligned}
$$

where $R_{0}=\sum_{i=0}^{N} \mathbf{1}\left(S_{i} \leq S_{0}\right)$ is the rank of $S_{0}$ when the $N+1$ variables $S_{0}, S_{1}, \ldots, S_{N}$ are ranked 
in nondecreasing order. Using (2.15) and Lemma 2.1, it then follows that

$$
\mathrm{P}\left[\hat{G}_{N}\left(S_{0}\right) \leq \alpha_{1}\right]=\mathrm{P}\left[\frac{N+1-R_{0}}{N} \leq \alpha_{1}\right]=\mathrm{P}\left[\frac{R_{0}}{N+1} \geq \frac{\left(1-\alpha_{1}\right) N+1}{N+1}\right]=\frac{I\left[\alpha_{1} N\right]+1}{N+1}
$$

for $0 \leq \alpha_{1} \leq 1$. Furthermore, $\hat{F}_{N}\left(S_{0}\right)=1-\hat{G}_{N}\left(S_{0}\right)$ with probability 1 , and (2.17) follows. We then get (2.18) on observing that: $\hat{F}_{N}(y) \geq q \Longleftrightarrow y \geq \hat{F}_{N}^{-1}(q)$ for $y \in \mathbb{R}$ and $0<q<1$ [see Reiss (1989, Appendix 1)]. Finally, to obtain (2.19), we note that

$$
\hat{p}_{N}\left(S_{0}\right) \equiv \frac{N \hat{G}_{N}\left(S_{0}\right)+1}{N+1} \leq \alpha \Longleftrightarrow \hat{G}_{N}\left(S_{0}\right) \leq \frac{\alpha(N+1)-1}{N}
$$

hence, since that $0 \leq \hat{G}_{N}\left(S_{0}\right) \leq 1$ and using (2.17),

$$
\begin{aligned}
\mathrm{P}\left[\hat{p}_{N}\left(S_{0}\right) \leq \alpha\right] & =\mathrm{P}\left[\hat{G}_{N}\left(S_{0}\right) \leq \frac{\alpha(N+1)-1}{N}\right] \\
& = \begin{cases}0, & \text { if } \alpha<1 /(N+1), \\
\frac{I[\alpha(N+1)-1]+1}{N+1}=\frac{I[\alpha(N+1)]}{N+1}, & \text { if } \frac{1}{N+1} \leq \alpha \leq 1, \\
1, & \text { if } \alpha>1,\end{cases}
\end{aligned}
$$

from which (2.19) follows on observing that $I[\alpha(N+1)]=0$ for $0 \leq \alpha<1 /(N+1)$.

Proof of Lemma 2.3 From (2.23) and the continuity of the $U(0,1)$ distribution, we see easily that $\mathrm{P}\left[\left(y_{i}, U_{i}\right)=\left(y_{j}, U_{j}\right)\right] \leq \mathrm{P}\left[U_{i}=U_{j}\right]=0$, for $i \neq j$, from which it follows that $\mathrm{P}\left[\tilde{R}_{i}=\tilde{R}_{j}\right]=0$ for $i \neq j$ and the rank vector $\left(\tilde{R}_{1}, \tilde{R}_{2}, \ldots, \tilde{R}_{N}\right)$ is with probability 1 a random permutation of the integers $(1,2, \ldots, N)$. Set $V_{i}=\left(y_{i}, U_{i}\right), i=1, \ldots, N$. By considering all possible permutations $\left(V_{r_{1}}, V_{r_{2}}, \ldots, V_{r_{N}}\right)$ of $\left(V_{1}, V_{2}, \ldots, V_{N}\right)$, and since $\left(V_{r_{1}}, V_{r_{2}} \ldots, V_{r_{N}}\right) \sim$ $\left(V_{1}, V_{2}, \ldots, V_{N}\right)$ for all permutations $\left(r_{1}, r_{2}, \ldots, r_{N}\right)$ of $(1,2, \ldots, N)$ [by the exchangeability assumption], the elements of $\left(\tilde{R}_{1}, \tilde{R}_{2}, \ldots, \tilde{R}_{N}\right)$ are also exchangeable. The result then follows from Lemma 2.1. The reader may note that an alternative proof could be obtained by modifying the proof of Theorem 29A of Hájek (1969) to relax the independence assumption for $y_{1}, \ldots, y_{N}$.

Proof of Proposition 2.4 Since the pairs $\left(S_{i}, U_{i}\right), i=0,1, \ldots, N$, are all distinct with probability 1 , we have almost surely:

$$
\begin{aligned}
\tilde{G}_{N}\left(S_{0}\right) & =\frac{1}{N} \sum_{i=1}^{N} \mathbf{1}\left[\left(S_{i}, U_{i}\right) \geq\left(S_{0}, U_{0}\right)\right]=1-\frac{1}{N} \sum_{i=1}^{N} \mathbf{1}\left[\left(S_{i}, U_{i}\right) \leq\left(S_{0}, U_{0}\right)\right] \\
& =1-\frac{1}{N}\left\{-1+\sum_{i=0}^{N} \mathbf{1}\left[\left(S_{i}, U_{i}\right) \leq\left(S_{0}, U_{0}\right)\right]\right\}=\left(N+1-\tilde{R}_{0}\right) / N
\end{aligned}
$$

where $\tilde{R}_{0}=\sum_{i=0}^{N} \mathbf{1}\left[\left(S_{i}, U_{i}\right) \leq\left(S_{0}, U_{0}\right)\right]$ is the randomized rank of $S_{0}$ obtained when ranking in 
ascending order [according to (2.23)] the $N+1$ pairs $\left(S_{i}, U_{i}\right), i=0,1, \ldots N$. Using Lemma 2.3, it follows that

$$
\begin{aligned}
\mathrm{P}\left[\tilde{G}_{N}\left(S_{0}\right) \leq \alpha_{1}\right] & =\mathrm{P}\left[\left(N+1-\tilde{R}_{0}\right) / N \leq \alpha_{1}\right]=\mathrm{P}\left[\frac{\tilde{R}_{0}}{N+1} \geq \frac{\left(1-\alpha_{1}\right) N+1}{N+1}\right] \\
& = \begin{cases}0, & \text { if } \alpha_{1}<0, \\
\frac{I\left[\alpha_{1} N\right]+1}{N+1}, & \text { if } 0 \leq \alpha_{1} \leq 1, \\
1, & \text { if } \alpha_{1}>1 .\end{cases}
\end{aligned}
$$

Since the pairs $\left(S_{i}, U_{i}\right), i=0,1, \ldots, N$, are all distinct with probability 1 , we also have $\tilde{F}_{N}\left(S_{0}\right)=1-\tilde{G}_{N}\left(S_{0}\right)$ with probability 1 , hence using the inequalities $(2.36)-(2.33)$,

$$
\mathrm{P}\left[\hat{G}_{N}\left(S_{0}\right) \leq \alpha_{1}\right] \leq \mathrm{P}\left[\tilde{G}_{N}\left(S_{0}\right) \leq \alpha_{1}\right]=\mathrm{P}\left[\tilde{F}_{N}\left(S_{0}\right) \geq 1-\alpha_{1}\right] \leq \mathrm{P}\left[\hat{F}_{N}\left(S_{0}\right) \geq 1-\alpha_{1}\right]
$$

and (2.36) is established. The identity $\mathrm{P}\left[\hat{F}_{N}\left(S_{0}\right) \geq 1-\alpha_{1}\right]=\mathrm{P}\left[S_{0} \geq \hat{F}_{N}^{-1}\left(1-\alpha_{1}\right)\right]$ follows from the equivalence: $\hat{F}_{N}(y) \geq q \Longleftrightarrow y \geq \hat{F}_{N}^{-1}(q), \forall y \in \mathbb{R}, 0<q<1$. Finally, to obtain (2.37), we observe that

$$
\tilde{p}_{N}\left(S_{0}\right)=\frac{N \tilde{G}_{N}\left(S_{0}\right)+1}{N+1} \leq \alpha \Longleftrightarrow \tilde{G}_{N}\left(S_{0}\right) \leq \frac{\alpha(N+1)-1}{N}
$$

hence, using (2.36),

$$
\begin{aligned}
\mathrm{P}\left[\tilde{p}_{N}\left(S_{0}\right) \leq \alpha\right] & =\mathrm{P}\left[\tilde{G}_{N}\left(S_{0}\right) \leq \frac{\alpha(N+1)-1}{N}\right] \\
& = \begin{cases}0, & \text { if } \alpha<1 /(N+1) \\
\frac{I[\alpha(N+1)-1]+1}{N+1}=\frac{I[\alpha(N+1)]}{N+1}, & \text { if } \frac{1}{N+1} \leq \alpha \leq 1\end{cases}
\end{aligned}
$$

from which (2.19) follows on observing that $I[\alpha(N+1)]=0$ for $0 \leq \alpha \leq 1 /(N+1)$.

Proof of Proposition 4.1 Since

$$
\hat{G}_{N}\left[S_{0} \mid \theta\right] \geq 1-\hat{F}_{N}\left[S_{0} \mid \theta\right],
$$

we have

$$
\mathrm{P}\left[\sup \left\{\hat{G}_{N}\left[S_{0} \mid \theta\right]: \theta \in \Omega_{0}\right\} \leq \alpha_{1}\right] \leq \mathrm{P}\left[\inf \left\{\hat{F}_{N}\left[S_{0} \mid \theta\right]: \theta \in \Omega_{0}\right\} \geq 1-\alpha_{1}\right] .
$$

When $\theta_{0} \in \Omega_{0}$, it is also clear that: $\inf _{\theta \in \Omega_{0}} \hat{F}_{N}\left[S_{0} \mid \theta\right] \geq 1-\alpha_{1} \Rightarrow \hat{F}_{N}\left[S_{0} \mid \theta_{0}\right] \geq 1-\alpha_{1}$. Thus, using Proposition 2.2,

$$
\mathrm{P}\left[\inf \left\{\hat{F}_{N}\left[S_{0} \mid \theta\right]: \theta \in \Omega_{0}\right\} \geq 1-\alpha_{1}\right] \leq \mathrm{P}\left[\hat{F}_{N}\left[S_{0} \mid \theta_{0}\right] \geq 1-\alpha_{1}\right]=\frac{I\left[\alpha_{1} N\right]+1}{N+1} .
$$


Furthermore,

$$
\begin{gathered}
\inf _{\theta \in \Omega_{0}} \hat{F}_{N}\left[S_{0} \mid \theta\right] \geq 1-\alpha_{1} \Longleftrightarrow \hat{F}_{N}\left[S_{0} \mid \theta\right] \geq 1-\alpha_{1}, \forall \theta \in \Omega_{0} \\
\Longleftrightarrow S_{0} \geq \hat{F}_{N}^{-1}\left[1-\alpha_{1} \mid \theta\right], \forall \theta \in \Omega_{0} \Longleftrightarrow S_{0} \geq \sup _{\theta \in \Omega_{0}} \hat{F}_{N}^{-1}\left[1-\alpha_{1} \mid \theta\right]
\end{gathered}
$$

so that, using Proposition2.2,

$$
\begin{aligned}
\mathrm{P}\left[S_{0} \geq \sup \left\{\hat{F}_{N}^{-1}\left[1-\alpha_{1} \mid \theta_{0}\right]: \theta \in \Omega_{0}\right\}\right] & =\mathrm{P}\left[\inf \left\{\hat{F}_{N}\left[S_{0} \mid \theta\right]: \theta \in \Omega_{0}\right\} \geq 1-\alpha_{1}\right] \\
& \leq \frac{I\left[\alpha_{1} N\right]+1}{N+1}
\end{aligned}
$$

and (4.20) is established. (4.21) follows in the same way on observing that $\sup _{\theta \in \Omega_{0}} \tilde{p}_{N}\left[S_{0} \mid \theta\right] \leq$ $\sup _{\theta \in \Omega_{0}} \hat{p}_{N}\left[S_{0} \mid \theta\right]$ and

$$
\sup _{\theta \in \Omega_{0}} \tilde{p}_{N}\left[S_{0} \mid \theta\right] \leq \alpha \Rightarrow \tilde{p}_{N}\left[S_{0} \mid \theta_{0}\right] \leq \alpha, \text { when } \theta_{0} \in \Omega_{0}
$$

Proof OF Proposition 4.2 Using (2.32) - (2.33), we have:

$$
\begin{aligned}
1-\hat{F}_{N}\left[S_{0} \mid \theta\right] & \leq \tilde{G}_{N}\left[S_{0} \mid \theta\right] \leq \hat{G}_{N}\left[S_{0} \mid \theta\right], \forall \theta, \\
1-\hat{G}_{N}\left[S_{0} \mid \theta\right] & \leq \tilde{F}_{N}\left[S_{0} \mid \theta\right] \leq \hat{F}_{N}\left[S_{0} \mid \theta\right],
\end{aligned}
$$

hence

$$
\begin{aligned}
\sup _{\theta \in \Omega_{0}} \tilde{G}_{N}\left[S_{0} \mid \theta\right] & \leq \sup _{\theta \in \Omega_{0}} \hat{G}_{N}\left[S_{0} \mid \theta\right], \\
1-\sup _{\theta \in \Omega_{0}} \hat{G}_{N}\left[S_{0} \mid \theta\right] & =\inf _{\theta \in \Omega_{0}}\left\{1-\hat{G}_{N}\left[S_{0} \mid \theta\right]\right\} \leq \inf _{\theta \in \Omega_{0}} \tilde{F}_{N}\left[S_{0} \mid \theta\right], \\
\sup _{\theta \in \Omega_{0}} \tilde{p}_{N}\left[S_{0} \mid \theta\right] & \leq \sup _{\theta \in \Omega_{0}} \hat{p}_{N}\left[S_{0} \mid \theta\right] .
\end{aligned}
$$

Furthermore, when $\theta_{0} \in \Omega_{0}$,

$$
\begin{aligned}
& \sup _{\theta \in \Omega_{0}} \tilde{G}_{N}\left[S_{0} \mid \theta\right] \leq \alpha_{1} \Rightarrow \tilde{G}_{N}\left[S_{0} \mid \theta_{0}\right] \leq \alpha_{1}, \\
& \inf _{\theta \in \Omega_{0}} \tilde{F}_{N}\left[S_{0} \mid \theta\right] \geq 1-\alpha_{1} \Rightarrow \tilde{F}_{N}\left[S_{0} \mid \theta_{0}\right] \geq 1-\alpha_{1}, \\
& \sup _{\theta \in \Omega_{0}} \tilde{p}_{N}\left[S_{0} \mid \theta\right] \leq \alpha_{1} \Rightarrow \tilde{p}_{N}\left[S_{0} \mid \theta_{0}\right] \leq \alpha_{1},
\end{aligned}
$$


hence, using Proposition 2.4, for $0 \leq \alpha_{1} \leq 1$ and for $0 \leq \alpha \leq 1$,

$$
\begin{aligned}
\mathrm{P}\left[\sup \left\{\hat{G}_{N}\left[S_{0} \mid \theta\right]: \theta \in \Omega_{0}\right\} \leq \alpha_{1}\right] & \leq \mathrm{P}\left[\sup \left\{\tilde{G}_{N}\left[S_{0} \mid \theta\right]: \theta \in \Omega_{0}\right\} \leq \alpha_{1}\right] \\
& \leq \mathrm{P}\left[\tilde{G}_{N}\left[S_{0} \mid \theta_{0}\right] \leq \alpha_{1}\right]=\frac{I\left[\alpha_{1} N\right]+1}{N+1}, \\
\mathrm{P}\left[\sup \left\{\hat{G}_{N}\left[S_{0} \mid \theta\right]: \theta \in \Omega_{0}\right\} \leq \alpha_{1}\right] & \leq \mathrm{P}\left[\inf \left\{\tilde{F}_{N}\left[S_{0} \mid \theta\right]: \theta \in \Omega_{0}\right\} \geq 1-\alpha_{1}\right] \\
& \leq \mathrm{P}\left[\tilde{F}_{N}\left[S_{0} \mid \theta_{0}\right] \geq 1-\alpha_{1}\right]=\frac{I\left[\alpha_{1} N\right]+1}{N+1}, \\
\mathrm{P}\left[\sup \left\{\hat{p}_{N}\left[S_{0} \mid \theta\right]: \theta \in \Omega_{0}\right\} \leq \alpha\right] & \leq \mathrm{P}\left[\sup \left\{\tilde{p}_{N}\left[S_{0} \mid \theta\right]: \theta \in \Omega_{0}\right\} \leq \alpha\right] \\
& \leq \mathrm{P}\left[\tilde{p}_{N}\left[S_{0} \mid \theta_{0}\right] \leq \alpha\right]=\frac{I[\alpha(N+1)]}{N+1} .
\end{aligned}
$$

Proof of Proposition 5.1 Using arguments similar to the ones in the proof of Proposition 4.2 [see (A.1) - (A.2)], it is easy to see that

$$
\begin{aligned}
\mathrm{P}\left[\sup \left\{\hat{G}_{T N}\left[S_{T 0} \mid \theta\right]: \theta \in C_{T}\right\} \leq \alpha_{1}\right] & \leq \mathrm{P}\left[\inf \left\{\hat{F}_{T N}\left[S_{T 0} \mid \theta\right]: \theta \in C_{T}\right\} \geq 1-\alpha_{1}\right] \\
& =\mathrm{P}\left[S_{T 0} \geq \sup \left\{\hat{F}_{T N}^{-1}\left[1-\alpha_{1} \mid \theta\right]: \theta \in C_{T}\right\}\right] .
\end{aligned}
$$

Further

$$
\begin{aligned}
& \mathrm{P}\left[\inf \left\{\hat{F}_{T N}\left[S_{T 0} \mid \theta\right]: \theta \in C_{T}\right\} \geq 1-\alpha_{1}\right] \\
& \quad=\mathrm{P}\left[\inf \left\{\hat{F}_{T N}\left[S_{T 0} \mid \theta\right]: \theta \in C_{T}\right\} \geq 1-\alpha_{1} \text { and } \theta_{0} \in C_{T}\right] \\
& \quad+\mathrm{P}\left[\inf \left\{\hat{F}_{T N}\left[S_{T 0} \mid \theta\right]: \theta \in C_{T}\right\} \geq 1-\alpha_{1} \text { and } \theta_{0} \notin C_{T}\right] \\
& \quad \leq \mathrm{P}\left[\hat{F}_{T N}\left[S_{T 0} \mid \theta_{0}\right] \geq 1-\alpha_{1}\right]+\mathrm{P}\left[\theta_{0} \notin C_{T}\right]=\frac{I\left[\alpha_{1} N\right]+1}{N+1}+\mathrm{P}\left[\theta_{0} \notin C_{T}\right]
\end{aligned}
$$

where the last identity follows from Proposition 2.2, hence, since $\lim _{T \rightarrow \infty} \mathrm{P}\left[\theta_{0} \notin C_{T}\right]=0$,

$$
\begin{aligned}
\lim _{T \rightarrow \infty} \mathrm{P}\left[\inf \left\{\hat{F}_{T N}\left[S_{T 0} \mid \theta\right]: \theta \in C_{T}\right\} \geq 1-\alpha_{1}\right] & \leq \frac{I\left[\alpha_{1} N\right]+1}{N+1}+\lim _{T \rightarrow \infty} \mathrm{P}\left[\theta_{0} \notin C_{T}\right] \\
& =\frac{I\left[\alpha_{1} N\right]+1}{N+1},
\end{aligned}
$$

.from which (5.9) and (5.10) follow.

Proof of Proposition 5.2 The result follows from arguments similar to the ones used in the proofs of Propositions 4.1 and $\mathbf{5 . 2}$ (with $\Omega_{0}$ replaced by $C_{T}$ ). 
In order to prove Proposition 6.1, it will be convenient to first demonstrate the following two lemmas

Lemma A.1 Continuity of $p$-VAlue function. Under the assumptions and notations (5.1), (5.2), (5.4), (5.5), (5.14) - (5.16) and (6.1), set

$$
\begin{aligned}
& Q_{T N}\left(\theta, x, u_{0}, \alpha_{1}\right)=\mathrm{P}\left[\tilde{G}_{T N}[x \mid \theta] \leq \alpha_{1} \mid U_{0}=u_{0}\right], \\
& \bar{Q}_{T N}\left(\theta, x, \alpha_{1}\right)=\mathrm{P}\left[\hat{G}_{T N}[x \mid \theta] \leq \alpha_{1}\right], 0 \leq \alpha_{1} \leq 1,
\end{aligned}
$$

and suppose $U_{0}$ is independent of $S_{T}(N, \theta)$. For any $\theta, \theta_{0} \in \Omega, x \in \mathbb{R}$ and $u_{0}, \alpha_{1} \in[0,1]$, the inequality

$$
\left|F_{T}[y \mid \theta]-F_{T}\left[y \mid \theta_{0}\right]\right| \leq \varepsilon, \forall y \in(x-\delta, x+\delta),
$$

where $\delta>0$, entails the inequalities:

$$
\begin{aligned}
\left|Q_{T N}\left(\theta, x, u_{0}, \alpha_{1}\right)-Q_{T N}\left(\theta_{0}, x, u_{0}, \alpha_{1}\right)\right| & \leq 3 C\left(N, \alpha_{1}\right) \varepsilon \\
\left|\bar{Q}_{T N}\left(\theta, x, \alpha_{1}\right)-\bar{Q}_{T N}\left(\theta_{0}, x, u_{0}\right)\right| & \leq 3 C\left(N, \alpha_{1}\right) \varepsilon
\end{aligned}
$$

where $C\left(N, \alpha_{1}\right)=N \sum_{k=0}^{I\left[\alpha_{1} N\right]}\left(\begin{array}{l}N \\ k\end{array}\right)$.

Proof. It is easy to see [as in (3.3)] that

$$
\begin{aligned}
Q_{T N}\left(\theta, x, u_{0}, \alpha_{1}\right) & =\mathrm{P}\left[\tilde{G}_{T N}(x \mid \theta) \leq \alpha_{1} \mid U_{0}=u_{0}\right] \\
& =\sum_{k=0}^{I\left[\alpha_{1} N\right]}\left(\begin{array}{l}
N \\
k
\end{array}\right) \bar{G}_{T}\left(x, u_{0} \mid \theta\right)^{k}\left[1-\bar{G}_{T}\left(x, u_{0} \mid \theta\right)\right]^{N-k}
\end{aligned}
$$

where

$$
\begin{aligned}
\bar{G}_{T}\left(x, u_{0} \mid \theta\right) & =\mathrm{P}\left(\mathbf{1}\left[\left(S_{T i}(\theta), U_{i}\right) \geq\left(x, u_{0}\right)\right]=1\right) \\
& =\mathrm{P}\left[S_{T i}(\theta)>x\right]+\mathrm{P}\left[S_{T i}(\theta)=x\right] \mathrm{P}\left[U_{i} \geq u_{0}\right] \\
& =1-F_{T}[x \mid \theta]+g_{T}(x \mid \theta)\left(1-u_{0}\right), 1 \leq i \leq T
\end{aligned}
$$

Note also that $g_{T}(x \mid \theta)=F_{T}[x \mid \theta]-\lim _{\delta_{0} \rightarrow 0^{+}} F_{T}\left[y-\delta_{0} \mid \theta\right]$. Then the inequality

$$
\left|F_{T}[y \mid \theta]-F_{T}\left[y \mid \theta_{0}\right]\right| \leq \varepsilon, \forall y \in(x-\delta, x+\delta),
$$

entails the following inequalities:

$$
\begin{array}{r}
\left|1-F_{T}[x \mid \theta]-1+F_{T}\left[x \mid \theta_{0}\right]\right| \leq \varepsilon, \\
\left|g_{T}(x \mid \theta)-g_{T}\left(x \mid \theta_{0}\right)\right|=\mid\left\{1-F_{T}[x \mid \theta]\right\}-\left\{1-F_{T}\left[x \mid \theta_{0}\right]\right\}
\end{array}
$$




$$
\begin{gathered}
+\lim _{\delta_{0} \rightarrow 0^{+}}\left\{F_{T}\left[y-\delta_{0} \mid \theta\right]-F_{T}\left[y-\delta_{0} \mid \theta_{0}\right]\right\} \mid \\
\leq\left|F_{T}[x \mid \theta]-F_{T}\left[x \mid \theta_{0}\right]\right|+\lim _{\delta_{0} \rightarrow 0^{+}}\left|F_{T}\left[y-\delta_{0} \mid \theta\right]-F_{T}\left[y-\delta_{0} \mid \theta_{0}\right]\right| \leq 2 \varepsilon,
\end{gathered}
$$

hence, for all $u_{0} \in[0,1]$,

$$
\begin{aligned}
\left|\bar{G}_{T}\left(x, u_{0} \mid \theta\right)-\bar{G}_{T}\left(x, u_{0} \mid \theta_{0}\right)\right| \leq & \left|F_{T}[x \mid \theta]-F_{T}\left[x \mid \theta_{0}\right]\right| \\
& +\left|1-u_{0}\right|\left|g_{T}(x \mid \theta)-g_{T}\left(x \mid \theta_{0}\right)\right| \leq 3 \varepsilon, \forall u_{0} \in[0,1], \\
\mid Q_{T N}\left(\theta, x, u_{0}, \alpha_{1}\right) \leq & Q_{T N}\left(\theta_{0}, x, u_{0}, \alpha_{1}\right) \mid \\
\leq & \mid \sum_{k=0}^{\left[N \alpha_{1}\right]}\left\{\begin{array}{l}
N \\
k
\end{array}\right) \bar{G}_{T}\left(x, u_{0} \mid \theta\right)^{k}\left[1-\bar{G}_{T}\left(x, u_{0} \mid \theta\right)\right]^{N-k} \\
& \left.-\bar{G}_{T}\left(x, u_{0} \mid \theta_{0}\right)^{k}\left[1-\bar{G}_{T}\left(x, u_{0} \mid \theta_{0}\right)\right]^{N-k}\right\} \mid \\
\leq & \sum_{k=0}^{\left[N \alpha_{1}\right]}\left(\begin{array}{l}
N \\
k
\end{array}\right)\left|\bar{G}_{T}\left(x, u_{0} \mid \theta\right)^{k}-\bar{G}_{T}\left(x, u_{0} \mid \theta_{0}\right)^{k}\right| \\
& +\left|\left[1-\bar{G}_{T}\left(x, u_{0} \mid \theta\right)\right]^{N-k}-\left[1-\bar{G}_{T}\left(x, u_{0} \mid \theta_{0}\right)\right]^{N-k}\right| \\
\leq & \sum_{k=0}^{\left[N \alpha_{1}\right]}\left\{\left(\begin{array}{l}
N \\
k
\end{array}\right) k\left|\bar{G}_{T}\left(x, u_{0} \mid \theta\right)-\bar{G}_{T}\left(x, u_{0} \mid \theta_{0}\right)\right|\right. \\
& \left.+(N-k)\left|\bar{G}_{T}\left(x, u_{0} \mid \theta\right)-\bar{G}_{T}\left(x, u_{0} \mid \theta_{0}\right)\right|\right\} \\
= & C\left(N, \alpha_{1}\right)\left|\bar{G}_{T}\left(x, u_{0} \mid \theta\right)-\bar{G}_{T}\left(x, u_{0} \mid \theta_{0}\right)\right| \leq 3 C\left(N, \alpha_{1}\right) \varepsilon,
\end{aligned}
$$

where $C\left(N, \alpha_{1}\right)=N \sum_{k=0}^{I\left[\alpha_{1} N\right]}\left(\begin{array}{l}N \\ k\end{array}\right)$, from which (A.5) follows. The inequality (A.6) follows in a similar way on noting that $\bar{Q}_{T N}\left(\theta, x, \alpha_{1}\right)=\sum_{k=0}^{I\left[\alpha_{1} N\right]}\left(\begin{array}{c}N \\ k\end{array}\right) G_{T}(x \mid \theta)^{k}\left[1-G_{T}(x \mid \theta)\right]^{N-k}$, where $G_{T}(x \mid \theta)=\mathrm{P}_{\theta}\left[S_{T i}(\theta) \geq x\right], 1 \leq i \leq N$.

Lemma A.2 COnVERgence of Bootstrap p-VAlues. Under the assumptions and notations of Lemma A.1, suppose that (6.2) and (6.3) also hold. If $\hat{\theta}_{T} \underset{T \rightarrow \infty}{\longrightarrow} \theta_{0}$ in probability and condition (6.4) or (6.5) - (6.7) holds, then

$$
\begin{gathered}
\sup _{0 \leq u_{0} \leq 1}\left|Q_{T N}\left(\hat{\theta}_{T}, S_{T 0}, u_{0}, \alpha_{1}\right)-Q_{T N}\left(\theta_{0}, S_{T 0}, u_{0}, \alpha_{1}\right)\right| \underset{T \rightarrow \infty}{\stackrel{p}{\longrightarrow}} 0, \\
\bar{Q}_{T N}\left(\hat{\theta}_{T}, S_{T 0}, \alpha_{1}\right)-\bar{Q}_{T N}\left(\theta_{0}, S_{T 0}, \alpha_{1}\right) \underset{T \rightarrow \infty}{\stackrel{p}{\longrightarrow}} 0 .
\end{gathered}
$$


We can now prove the following proposition.

Proof. Let $\alpha_{1} \in[0,1], \varepsilon>0$ and $\varepsilon_{0}>0$ and suppose first that (6.4) holds. Then, using Lemma A.1, we can find $\delta>0$ and $T_{1}$ such that

$$
\begin{aligned}
x \in D_{T 0}\left(\varepsilon_{0}\right), & \left\|\theta-\theta_{0}\right\| \leq \delta \text { and } T>T_{1} \\
& \Rightarrow\left|F_{T}[x \mid \theta]-F_{T}\left[x \mid \theta_{0}\right]\right| \leq \varepsilon_{1} \equiv \varepsilon /\left[3 C\left(N, \alpha_{1}\right)\right] \\
& \Rightarrow\left|Q_{T N}\left(\theta, x, u_{0}, \alpha_{1}\right)-Q_{T N}\left(\theta_{0}, x, u_{0}, \alpha_{1}\right)\right| \leq \varepsilon, \forall u_{0} \in[0,1] .
\end{aligned}
$$

Thus

$$
S_{T 0} \in D_{T 0}\left(\varepsilon_{0}\right) \text { and }\left\|\hat{\theta}_{T}-\theta_{0}\right\| \leq \delta \Rightarrow \Delta_{T N}\left(\hat{\theta}_{T}, \theta_{0}, S_{T 0}, \alpha_{1}\right) \leq \varepsilon
$$

where $\Delta_{T N}\left(\hat{\theta}_{T}, \theta_{0}, S_{T 0}, \alpha_{1}\right) \equiv \sup _{0 \leq u_{0} \leq 1}\left|Q_{T N}\left(\hat{\theta}_{T}, S_{T 0}, u_{0}, \alpha_{1}\right)-Q_{T N}\left(\theta_{0}, x, u_{0}, \alpha_{1}\right)\right|$, hence

$$
\begin{aligned}
\mathrm{P}\left[\Delta_{T N}\left(\hat{\theta}_{T}, \theta_{0}, S_{T 0}, \alpha_{1}\right) \leq \varepsilon\right] & \geq \mathrm{P}\left[S_{T 0} \in D_{T 0}\left(\varepsilon_{0}\right) \text { and }\left\|\hat{\theta}_{T}-\theta_{0}\right\| \leq \delta\right] \\
& \geq 1-\mathrm{P}\left[S_{T 0} \notin D_{T 0}\left(\varepsilon_{0}\right)\right]-\mathrm{P}\left[\left\|\hat{\theta}_{T}-\theta_{0}\right\|>\delta\right] \\
& =\mathrm{P}\left[S_{T 0} \in D_{T 0}\left(\varepsilon_{0}\right)\right]-\mathrm{P}\left[\left\|\hat{\theta}_{T}-\theta_{0}\right\|>\delta\right]
\end{aligned}
$$

Since $\hat{\theta}_{T} \stackrel{p}{\rightarrow} \theta_{0}$, it follows that

$$
\liminf _{T \rightarrow \infty} \mathrm{P}\left[\Delta_{T N}\left(\hat{\theta}_{T}, \theta_{0}, S_{T 0}, \alpha_{1}\right) \leq \varepsilon\right] \geq \liminf _{T \rightarrow \infty} \mathrm{P}\left[S_{T 0} \in D_{T 0}\left(\varepsilon_{0}\right)\right] \geq 1-\varepsilon_{0}
$$

for any $\varepsilon_{0}>0$, hence $\lim _{T \rightarrow \infty} \mathrm{P}\left[\Delta_{T N}\left(\hat{\theta}_{T}, \theta_{0}, S_{T 0}, \alpha_{1}\right) \leq \varepsilon\right]=1$. Since the latter identity holds for any $\varepsilon>0$, (A.7) is established. (A.8) follows in a similar way upon using (A.6).

Suppose now (6.5) - (6.7) hold instead of (6.4). Then, $\left(S_{T 0}, \hat{\theta}_{T}\right) \underset{T \rightarrow \infty}{\stackrel{p}{\longrightarrow}}\left(S_{0}, \theta_{0}\right)$ and

$$
\left(S_{\bar{T}_{k}}, \hat{\theta}_{\bar{T}_{k}}\right) \underset{T \rightarrow \infty}{\stackrel{p}{\longrightarrow}}\left(S_{0}, \theta_{0}\right)
$$

for any subsequence $\left\{\left(S_{\bar{T}_{k}}, \hat{\theta}_{\bar{T}_{k}}\right): k=1,2, \ldots\right\}$ of $\left\{\left(S_{T}, \hat{\theta}_{T}\right): T \geq I_{0}\right\}$. Since $S_{T 0}$ and $\hat{\theta}_{T}$, $T \geq I_{0}$, are random variables (or vectors) defined on $\mathcal{Z}$, we can write $S_{T 0}=S_{T 0}(\omega), \hat{\theta}_{T}=\hat{\theta}_{T}(\omega)$ and $S_{0}=S_{0}(\omega), \omega \in \mathcal{Z}$. By (6.6), the event

$$
A_{0}=\left\{\omega: S_{0}(\omega) \in D_{0} \text { and } S_{T 0}(\omega) \in D_{0}, \text { for } T \geq I_{0}\right\}
$$

has probability one. Furthermore, by (A.9), the subsequence $\left(\left(S_{\bar{T}_{k}}, \hat{\theta}_{\bar{T}_{k}}^{\prime}\right)^{\prime}\right.$ contains a further subsequence $\left(S_{T_{k} 0}, \hat{\theta}_{T_{k}}^{\prime}\right)^{\prime}, k \geq 1$ such that $\left(S_{T_{k} 0}, \hat{\theta}_{T_{k}}^{\prime}\right)^{\prime} \underset{T \rightarrow \infty}{\longrightarrow}\left(S_{0}, \theta_{0}^{\prime}\right)^{\prime}$ a.s. (where $\left.T_{1}<T_{2}<\cdots\right)$; see Bierens (1994, pp. 22-23). Consequently, the set

$$
C_{0}=\left\{\omega \in \mathcal{Z}: S_{0}(\omega) \in D_{0}, \lim _{k \rightarrow \infty} S_{T_{k} 0}(\omega)=S_{0}(\omega) \text { and } \lim _{k \rightarrow \infty} \hat{\theta}_{T_{k}}(\omega)=\theta_{0}\right\}
$$


has probability one. Now, let $\varepsilon>0$. By (6.7), for any $x \in D_{0}$, we can find $\delta(x, \varepsilon)>0$, $T(x, \varepsilon)>0$ and an open neighborhood $B(x, \varepsilon)$ of $x$ such that

$$
\left\|\theta-\theta_{0}\right\| \leq \delta(x, \varepsilon) \text { and } T>T(x, \varepsilon) \Rightarrow\left|F_{T}[y \mid \theta]-F_{T}\left[y \mid \theta_{0}\right]\right| \leq \varepsilon, \forall y \in B(x, \varepsilon) \cap D_{0} .
$$

Furthermore, for $\omega \in C_{0}$, we can find $k_{0}$ such that

$$
k \geq k_{0} \Rightarrow S_{T_{k} 0}(\omega) \in B\left(S_{0}(\omega), \varepsilon\right) \cap D_{0} \text { and }\left\|\hat{\theta}_{T_{k}}-\theta_{0}\right\| \leq \delta\left(S_{0}(\omega), \varepsilon\right),
$$

so that $T_{k}>\max \left\{T\left(S_{0}(\omega), \varepsilon\right), T_{k_{0}}\right\}$ entails $\left|F_{T_{k}}\left[S_{T_{k} 0}(\omega) \mid \theta_{T_{k}}(\omega)\right]-F_{T_{k}}\left[S_{T_{k} 0}(\omega) \mid \theta_{0}\right]\right| \leq$ $\varepsilon$. Thus $\lim _{k \rightarrow \infty}\left\{F_{T_{k}}\left[S_{T_{k} 0}(\omega) \mid \theta_{T_{k}}(\omega)\right]-F_{T_{k}}\left[S_{T_{k} 0}(\omega) \mid \theta_{0}\right]\right\}=0$ for $\omega \in C_{0}$, hence, using Lemma A.1, $\lim _{k \rightarrow \infty} \Delta_{T_{k} N}\left(\hat{\theta}_{T_{k}}(\omega), \theta_{0}, S_{T_{k} 0}(\omega), \alpha_{1}\right)=0$ and $\Delta_{T_{k} N}\left(\hat{\theta}_{T_{k}}, \theta_{0}, S_{T_{k} 0}, \alpha_{1}\right) \underset{k \rightarrow 0}{\longrightarrow} 0$, a.s. This shows that any subsequence of the sequence $\Delta_{T N}\left(\hat{\theta}_{T}, \theta_{0}, S_{T 0}, \alpha_{1}\right), T \geq I_{0}$, contains a further subsequence which converge a.s. to zero. It follows that $\Delta_{T N}\left(\hat{\theta}_{T}, \theta_{0}, S_{T 0}, \alpha_{1}\right) \underset{T \rightarrow \infty}{\stackrel{p}{\longrightarrow}} 0$ and (A.7) is established. The proof of (A.8) under the condition (6.5) - (6.7) is similar.

Proof of Proposition 6.1 Using the fact that $\hat{\theta}_{T}, S_{T 0}$ and $U_{0}$ are independent of $S_{T}(N, \theta)$, we can write

$$
\begin{aligned}
\mathrm{P}\left[\tilde{G}_{T N}\left[S_{T 0} \mid \hat{\theta}_{T}\right] \leq \alpha_{1}\right]-\mathrm{P}\left[\tilde{G}_{T N}\left[S_{T 0} \mid \theta_{0}\right] \leq \alpha_{1}\right] \\
=\mathrm{E}\left\{\mathrm{P}\left[\tilde{G}_{T N}\left[S_{T 0} \mid \hat{\theta}_{T}\right] \leq \alpha_{1} \mid\left(\hat{\theta}_{T}, S_{T 0}, U_{0}\right)\right]\right. \\
\left.\quad-\mathrm{P}\left[\tilde{G}_{T N}\left[S_{T 0} \mid \theta_{0}\right] \leq \alpha_{1} \mid\left(\hat{\theta}_{T}, S_{T 0}, U_{0}\right)\right]\right\} \\
=\mathrm{E}\left[Q_{T N}\left(\hat{\theta}_{T}, S_{T 0}, U_{0}, \alpha_{1}\right)-Q_{T N}\left(\theta_{0}, S_{T 0}, U_{0}, \alpha_{1}\right)\right] .
\end{aligned}
$$

From Lemma A.1 and using the Lebesgue dominated convergence theorem, we then get

$$
\begin{aligned}
\left|\mathrm{P}\left[\tilde{G}_{T N}\left[S_{T 0} \mid \hat{\theta}_{T}\right] \leq \alpha_{1}\right]-\mathrm{P}\left[\tilde{G}_{T N}\left[S_{T 0} \mid \theta_{0}\right] \leq \alpha_{1}\right]\right| \\
\quad=\left|\mathrm{E}\left[Q_{T N}\left(\hat{\theta}_{T}, S_{T 0}, U_{0}, \alpha_{1}\right)-Q_{T N}\left(\theta_{0}, S_{T 0}, U_{0}, \alpha_{1}\right)\right]\right| \\
\quad \leq \mathrm{E}\left\{\left|Q_{T N}\left(\hat{\theta}_{T}, S_{T 0}, U_{0}, \alpha_{1}\right)-Q_{T N}\left(\theta_{0}, S_{T 0}, U_{0}, \alpha_{1}\right)\right|\right\} \\
\left.\quad \leq \mathrm{E}\left[\sup _{0 \leq u_{0} \leq 1} \mid Q_{T N}\left(\hat{\theta}_{T}, S_{T 0}, u_{0}, \alpha_{1}\right)-Q_{T N}\left(\theta_{0}, S_{T 0}, u_{0}, \alpha_{1}\right)\right]\right]_{T \rightarrow \infty}^{\longrightarrow} 0 .
\end{aligned}
$$

We can show in a similar way that

$$
\left|\mathrm{P}\left[\hat{G}\left[S_{T 0} \mid \hat{\theta}_{T}\right] \leq \alpha_{1}\right]-\mathrm{P}\left[G\left[S_{T 0} \mid \theta_{0}\right] \leq \alpha_{1}\right]\right| \underset{T \rightarrow \infty}{\longrightarrow} 0
$$

from which we get (6.8). (6.9) then follows from the definitions of $\tilde{p}_{T N}(x \mid \theta)$ and $\hat{p}_{T N}(x \mid \theta)$. 


\section{References}

Andrews, D. W. K. (2000), 'Inconsistency of the bootstrap when a parameter is on the boundary of the parameter space', Econometrica 68, 399-405.

Athreya, K. B. (1987), 'Bootstrap of the mean in the infinite variance case', The Annals of Statistics 15, 724-731.

Barnard, G. A. (1963), 'Comment on 'The spectral analysis of point processes' by M. S. Bartlett', Journal of the Royal Statistical Society, Series B 25, 294.

Basawa, I. V., Mallik, A. K., McCormick, W. P., Reeves, J. H. and Taylor, R. L. (1991), 'Bootstrapping unstable first-order autoregressive processes', The Annals of Statistics 19, 1098-1101.

Benkwitz, A., Lütkepohl, H. and Neumann, M. H. (2000), 'Problems related to confidence intervals for impulse responses of autoregressive processes', Econometric Reviews 19, 69-103.

Beran, R. and Ducharme, G. R. (1991), Asymptotic Theory for Bootstrap Methods in Statistics, Centre de Recherches Mathématiques, Université de Montréal, Montréal, Canada.

Besag, J. and Diggle, P. J. (1977), 'Simple Monte Carlo tests for spatial pattern', Applied Statistics 26, 327-333.

Bierens, H. J. (1994), Topics in Advanced Econometrics, Cambridge University Press, New York.

Birnbaum, Z. W. (1974), Computers and unconventional test-statistics, in F. Proschan and R. J. Serfling, eds, 'Reliability and Biometry', SIAM, Philadelphia, PA, pp. 441-458.

Brown, L. D. and Purves, R. (1973), 'Measurable selections of extrema', The Annals of Statistics 1, 902-912.

Campbell, B. and Dufour, J.-M. (1997), 'Exact nonparametric tests of orthogonality and random walk in the presence of a drift parameter', International Economic Review 38, 151-173.

Chernick, M. R. (1999), Bootstrap Methods: A Practitioner's Guide, John Wiley \& Sons, New York.

Davison, A. and Hinkley, D. (1997), Bootstrap Methods and Their Application, Cambridge University Press, Cambridge (UK).

Debreu, G. (1967), Integration of correspondences, in L. LeCam and J. Neyman, eds, 'Proceedings of the Fifth Berkeley Symposium on Mathematical Statistics and Probability', Vol. II-1, The University of California Press, Berkeley and Los Angeles, California, pp. 351-372.

Dufour, J.-M. (1989), 'Nonlinear hypotheses, inequality restrictions, and non-nested hypotheses: Exact simultaneous tests in linear regressions', Econometrica 57, 335-355. 
Dufour, J.-M. (1990), 'Exact tests and confidence sets in linear regressions with autocorrelated errors', Econometrica 58, 475-494.

Dufour, J.-M., Farhat, A., Gardiol, L. and Khalaf, L. (1998), 'Simulation-based finite sample normality tests in linear regressions', The Econometrics Journal 1, 154-173.

Dufour, J.-M., Hallin, M. and Mizera, I. (1998), 'Generalized runs tests for heteroskedastic time series', Journal of Nonparametric Statistics 9, 39-86.

Dufour, J.-M. and Jasiak, J. (2001), 'Finite sample limited information inference methods for structural equations and models with generated regressors', International Economic Review 42, 815-843.

Dufour, J.-M. and Jouini, T. (2004), Finite-sample simulation-based tests in VAR models with applications to order selection and causality testing, Technical report, Centre interuniversitaire de recherche en analyse des organisations (CIRANO) and Centre interuniversitaire de recherche en économie quantitative (CIREQ), Université de Montréal.

Dufour, J.-M. and Khalaf, L. (2003a), Finite sample tests in seemingly unrelated regressions, in D. E. A. Giles, ed., 'Computer-Aided Econometrics', Marcel Dekker, New York, chapter 2, pp. 11-35.

Dufour, J.-M. and Khalaf, L. (2003b), Simulation-based finite-sample inference in simultaneous equations, Technical report, Centre interuniversitaire de recherche en analyse des organisations (CIRANO) and Centre interuniversitaire de recherche en économie quantitative (CIREQ), Université de Montréal.

Dufour, J.-M. and Kiviet, J. F. (1996), 'Exact tests for structural change in first-order dynamic models', Journal of Econometrics 70, 39-68.

Dufour, J.-M. and Kiviet, J. F. (1998), 'Exact inference methods for first-order autoregressive distributed lag models', Econometrica 66, 79-104.

Dufour, J.-M. and Valéry, P. (2004), A simple closed-form estimator for a stochastic volatility model, Technical report, Centre interuniversitaire de recherche en analyse des organisations (CIRANO) and Centre interuniversitaire de recherche en économie quantitative (CIREQ), Université de Montréal, Montréal, Canada.

Dwass, M. (1957), 'Modified randomization tests for nonparametric hypotheses', Annals of Mathematical Statistics 28, 181-187.

Edgington, E. S. (1980), Randomization Tests, Marcel Dekker, New York.

Edgington, E. S. (1995), Randomization Tests, 3rd Edition, Marcel Dekker, New York.

Edwards, D. (1985), 'Exact simulation-based inference: A survey, with additions', Journal of Statistical Computation and Simulation 22, 307-326. 
Edwards, D. and Berry, J. J. (1987), 'The efficiency of simulation-based multiple comparisons', Biometrics 43, 913-928.

Efron, B. (1982), The Jacknife, the Bootstrap and Other Resampling Plans, CBS-NSF Regional Conference Series in Applied Mathematics, Monograph No. 38, Society for Industrial and Applied Mathematics, Philadelphia, PA.

Efron, B. and Tibshirani, R. J. (1993), An Introduction to the Bootstrap, Vol. 57 of Monographs on Statistics and Applied Probability, Chapman \& Hall, New York.

Foutz, R. V. (1980), 'A method for constructing exact tests from test statistics that have unknown null distributions', Journal of Statistical Computation and Simulation 10, 187-193.

Gallant, A. R. and Tauchen, G. (1996), 'Which moments to match?', Econometric Theory 12, 657 -681 .

Goffe, W. L., Ferrier, G. D. and Rogers, J. (1994), 'Global optimization of statistical functions with simulated annealing', Journal of Econometrics 60, 65-99.

Good, P. (1994), Permutation Tests. A Practical Guide to Resampling Methods for Testing Hypotheses, second edn, Springer-Verlag, New York.

Gouriéroux, C. and Monfort, A. (1996), Simulation-Based Econometric Methods, Oxford University Press, Oxford, U.K.

Green, B. F. (1977), 'A practical interactive program for randomization tests of location', The American Statistician 31, 37-39.

Hájek, J. (1969), A Course in Nonparametric Statistics, Holden-Day, San Francisco.

Hajivassiliou, V. A. (1993), Simulation estimation methods for limited dependent variables, in (Maddala, Rao and Vinod 1993), pp. 519-543.

Hall, P. (1992), The Bootstrap and Edgeworth Expansion, Springer-Verlag, New York.

Hall, P. and Titterington, D. M. (1989), 'The effect of simulation order on level accuracy and power of Monte Carlo tests', Journal of the Royal Statistical Society, Series B 51, 459-467.

Hope, A. C. A. (1968), 'A simplified Monte Carlo test procedure', Journal of the Royal Statistical Society, Series B 30, 582-598.

Horowitz, J. L. (1997), Bootstrap methods in econometrics: Theory and numerical performance, in D. Kreps and K. W. Wallis, eds, 'Advances in Economics and Econometrics: Theory and Applications. Seventh World Congress. 3 Volumes', Cambridge University Press, Cambridge, U.K., pp. 188-222.

Inoue, A. and Kilian, L. (2002), 'Bootstrapping autoregressive processs with possible unit roots', Econometrica 70(1), 377-391. 
Inoue, A. and Kilian, L. (2003), 'The continuity of the limit distribution in the parameter of interest is not essential for the validity of the bootstrap', Econometric Theory 19(6), 944-961.

Jeong, J. and Maddala, G. S. (1993), A perspective on application of bootstrap methods in econometrics, in (Maddala et al. 1993), pp. 573-610.

Jöckel, K.-H. (1986), 'Finite sample properties and asymptotic efficiency of Monte Carlo tests', The Annals of Statistics 14, 336-347.

Jouneau-Sion, F. and Torrès, O. (2003), MMC techniques for limited dependent variables models: Implementation by the branch and bound algorithm, Technical report, Groupe d'étude en mathématiques appliquées à la recherche sociale, Université de Lille 3, Lille, France. Journal of Econometrics, forthcoming.

Keane, M. P. (1993), Simulation estimation for panel data models with limited dependent variables, in (Maddala et al. 1993), pp. 545-571.

Keller-McNulty, S. and Higgins, J. J. (1987), 'Effect of tail weight and outliers and type-I error of robust permutation tests for location', Communications in Statistics, Simulation and Computation 16(1), 17-35.

Kiviet, J. F. and Dufour, J.-M. (1997), 'Exact tests in single equation autoregressive distributed lag models', Journal of Econometrics 80, 325-353.

Lehmann, E. L. (1986), Testing Statistical Hypotheses, 2nd edition, John Wiley \& Sons, New York.

Lock, R. H. (1991), 'A sequential approximation to a permutation test', Communications in Statistics, Simulation and Computation 20(1), 341-363.

Maddala, G. S., Rao, C. R. and Vinod, H. D., eds (1993), Handbook of Statistics 11: Econometrics, North-Holland, Amsterdam.

Manly, B. F. J. (1997), Randomization, Bootstrap and Monte Carlo Methods in Biology; Permutation Test, second edn, Chapman \& Hall, London.

Mariano, R. S. and Brown, B. W. (1993), Stochastic simulation for inference in nonlinear errors-invariables models, in (Maddala et al. 1993), pp. 611-627.

Marriott, F. H. C. (1979), 'Barnard's Monte Carlo tests: How many simulations?', Applied Statistics 28, 75-77.

McFadden, D. (1989), 'A method of simulated moments for estimation of discrete response models without numerical integration', Econometrica 57, 995-1026.

Noreen, E. W. (1989), Computer Intensive Methods for Testing Hypotheses: An Introduction, John Wiley \& Sons, New York. 
Reiss, H. D. (1989), Approximate Distributions of Order Statistics with Applications to Nonparametric Statistics, Springer Series in Statistics, Springer-Verlag, New York.

Ripley, B. D. (1981), Spatial Statistics, John Wiley \& Sons, New York.

Shao, S. and Tu, D. (1995), The Jackknife and Bootstrap, Springer-Verlag, New York.

Shorack, G. R. and Wellner, J. A. (1986), Empirical Processes with Applications to Statistics, John Wiley \& Sons, New York.

Sriram, T. N. (1994), 'Invalidity of bootstrap for critical branching processes with immigration', The Annals of Statistics 22, 1013-1023.

Stinchcombe, M. B. and White, H. (1992), 'Some measurability results for extrema of random functions over random sets', Review of Economic Studies 59, 495-512.

Vadiveloo, J. (1983), 'On the theory of modified randomization tests for nonparametric hypotheses', Communications in Statistics, Theory and Methods 12(14), 1581-1596.

Vinod, H. D. (1993), Bootstrap methods: Applications in econometrics, in (Maddala et al. 1993), pp. 629-661. 